\title{
A POSTERIORI ERROR BOUNDS FOR REDUCED-BASIS APPROXIMATIONS OF PARAMETRIZED PARABOLIC PARTIAL DIFFERENTIAL EQUATIONS
}

\author{
Martin A. Grepl ${ }^{1}$ And Anthony T. Patera ${ }^{2}$
}

\begin{abstract}
In this paper, we extend the reduced-basis methods and associated a posteriori error estimators developed earlier for elliptic partial differential equations to parabolic problems with affine parameter dependence. The essential new ingredient is the presence of time in the formulation and solution of the problem - we shall "simply" treat time as an additional, albeit special, parameter. First, we introduce the reduced-basis recipe - Galerkin projection onto a space $W_{N}$ spanned by solutions of the governing partial differential equation at $N$ selected points in parameter-time space - and develop a new greedy adaptive procedure to "optimally" construct the parameter-time sample set. Second, we propose error estimation and adjoint procedures that provide rigorous and sharp bounds for the error in specific outputs of interest: the estimates serve a priori to construct our samples, and a posteriori to confirm fidelity. Third, based on the assumption of affine parameter dependence, we develop offlineonline computational procedures: in the offline stage, we generate the reduced-basis space; in the online stage, given a new parameter value, we calculate the reduced-basis output and associated error bound. The operation count for the online stage depends only on $N$ (typically small) and the parametric complexity of the problem; the method is thus ideally suited for repeated, rapid, reliable evaluation of input-output relationships in the many-query or real-time contexts.
\end{abstract}

Mathematics Subject Classification. 35K15, 65M15.

Received: October 13, 2004. Revised: December 6, 2004.

\section{INTRODUCTION}

The design, optimization, control, and characterization of engineering components or systems often requires repeated, reliable, and real-time prediction of performance metrics, or outputs - such as heat fluxes or flowrates. These outputs are typically functionals of field variables - such as temperatures or velocities - associated with a parametrized partial differential equation; the parameters, or inputs, serve to identify a particular configuration of the component. The relevant system behaviour is thus described by an implicit input-output relationship, evaluation of which demands solution of the underlying partial differential equation (PDE). Our goal is the development of numerical methods that permit the efficient and reliable evaluation of this PDE-induced inputoutput relationship in real-time or in the limit of many queries.

\footnotetext{
Keywords and phrases. Parabolic partial differential equations, diffusion equation, parameter-dependent systems, reduced-basis methods, output bounds, Galerkin approximation, a posteriori error estimation.

1 Massachusetts Institute of Technology, Room 3-264, Cambridge, MA, USA.

2 Massachusetts Institute of Technology, Room 3-266, Cambridge, MA, USA. patera@mit.edu (corresponding author).
} 
To achieve this goal we will pursue the reduced-basis method. The reduced-basis method was first introduced in the late 1970s for the nonlinear analysis of structures $[1,27,28]$ and has subsequently been further investigated and developed more broadly $[3,7,13,31,32,37]$. In the more recent past the reduced-basis approach and in particular associated a posteriori error estimation procedures have been successfully developed for (non)linear and (non)coercive elliptic PDEs with affine parameter dependence [23,34,41-43]; in this paper, we consider the extension of these methods to certain classes of parabolic PDEs with affine parameter dependence - time is the essential new ingredient.

Many model-order reduction techniques for time-dependent systems are proposed in the literature: the most well-known are proper orthogonal decomposition (POD or Karhunen-Loève decomposition) [40], balanced truncation [26], and various related hybrid [19,45] techniques; see also [20,33] for an application of the reducedbasis method to initial value problems. However, none of these frameworks accommodate parametric variation (see [10] for an exception) or a posteriori error estimation. The contributions here are thus $(i)$ the simultaneous dependence of the field variable (and output) on both time and parameters, and ( $i i)$ the introduction of rigorous a posteriori error estimators.

To motivate our approach we consider an important class of applications - optimal control - which requires repeated and often real-time evaluation of input-output relationships. If the dynamics are described by PDEs, the cost quickly becomes prohibitively large $[11,15,21]$, and hence reduced-order models (e.g., of the variety described above) are often employed: applications range from fluid flow [16-18, 36] to hyperthermia treatment $[24,25]$ to thermal processing of semiconductors [30] and canned foods [4]. To address this class of problems our approach must be able to rigorously treat $(a)$ control inputs that are not known a priori - often a problem within the model reduction context, and $(b)$ outputs, functionals of the time-dependent field variable, that are also (scalar) functions of time.

This paper is organized as follows: in Section 2 we introduce the necessary notation and state the problem. The reduced-basis approximation and computational considerations are discussed in Section 3 . In Section 4 we introduce rigorous and sharp a posteriori error bounds for the primal variable, dual variable, and output of interest. We propose an adaptive procedure to select the "optimal sampling set" in Section 5, and finally we present numerical results in Section 6.

\section{Problem statement}

\subsection{Abstract formulation}

We first define the Hilbert spaces $Y^{\mathrm{e}} \equiv H_{0}^{1}(\Omega)$ - or, more generally, $H_{0}^{1}(\Omega) \subset Y^{\mathrm{e}} \subset H^{1}(\Omega)-$ and $X^{\mathrm{e}} \equiv L^{2}(\Omega)$, where $H^{1}(\Omega)=\left\{v \mid v \in L^{2}(\Omega), \nabla v \in\left(L^{2}(\Omega)\right)^{d}\right\}, H_{0}^{1}(\Omega)=\left\{v\left|v \in H^{1}(\Omega), v\right|_{\partial \Omega}=0\right\}$, and $L^{2}(\Omega)$ is the space of square integrable functions over $\Omega$ [35]; here $\Omega$ is a bounded domain in $\boldsymbol{R}^{d}$ with Lipschitz continuous boundary $\partial \Omega$. The inner product and norm associated with $Y^{\mathrm{e}}\left(X^{\mathrm{e}}\right)$ are given by $(\cdot, \cdot)_{Y^{\mathrm{e}}}\left((\cdot, \cdot)_{X^{\mathrm{e}}}\right)$ and $\|\cdot\|_{Y^{\mathrm{e}}}=(\cdot, \cdot)_{Y^{\mathrm{e}}}^{1 / 2}$ $\left(\|\cdot\|_{X^{\mathrm{e}}}=(\cdot, \cdot)_{X^{\mathrm{e}}}^{1 / 2}\right)$, respectively; for example, $(w, v)_{Y^{\mathrm{e}}} \equiv \int_{\Omega} \nabla w \cdot \nabla v+\int_{\Omega} w v, \forall w, v \in Y^{\mathrm{e}}$, and $(w, v)_{X^{\mathrm{e}}} \equiv$ $\int_{\Omega} w v, \forall w, v \in X^{\mathrm{e}}$.

For simplicity, we directly consider a time-discrete framework associated to the time interval $\left.I \equiv] 0, t_{f}\right]$ $\left(\bar{I} \equiv\left[0, t_{f}\right]\right)$. We divide $\bar{I}$ into $K$ subintervals of equal length $\Delta t=\frac{t_{f}}{K}$ and define $t^{k} \equiv k \Delta t, 0 \leq k \leq K \equiv \frac{t_{f}}{\Delta t}$, and $\mathbb{I} \equiv\left\{t^{0}, \ldots, t^{k}\right\}$; for notational convenience, we also introduce $\mathbb{K} \equiv\{1, \ldots, K\}$. We shall consider EulerBackward for the time integration; we can also readily treat higher-order schemes such as Crank-Nicolson [14]. Clearly, our results must be stable as $\Delta t \rightarrow 0, K \rightarrow \infty$.

We may now introduce the "exact" (superscript e) - more precisely, semi-discrete - problem: given a parameter $\mu \in \mathcal{D} \subset \boldsymbol{R}^{P}$, we evaluate the (here, single) output of interest

$$
s^{\mathrm{e}}\left(\mu, t^{k}\right)=\ell\left(y^{\mathrm{e}}\left(\mu, t^{k}\right)\right), \quad \forall k \in \mathbb{K}
$$


where the field variable, $y^{\mathrm{e}}\left(\mu, t^{k}\right) \in Y^{\mathrm{e}}, \forall k \in \mathbb{K}$, satisfies the weak form of the $\mu$-parametrized parabolic PDE [5]

$$
m\left(y^{\mathrm{e}}\left(\mu, t^{k}\right), v ; \mu\right)+\Delta t a\left(y^{\mathrm{e}}\left(\mu, t^{k}\right), v ; \mu\right)=m\left(y^{\mathrm{e}}\left(\mu, t^{k-1}\right), v ; \mu\right)+\Delta t b(v ; \mu) u\left(t^{k}\right), \quad \forall v \in Y^{\mathrm{e}}, \forall k \in \mathbb{K},
$$

with initial condition (say) $y^{\mathrm{e}}\left(\mu, t^{0}\right)=y_{0}(\mu)=0$. Here $\mu$ and $\mathcal{D}$ are the input and input domain; $a(\cdot, \cdot ; \mu)$ is a $Y^{\mathrm{e}}$-continuous bilinear form; $m(\cdot, \cdot ; \mu)$ and $b(\cdot ; \mu), \ell(\cdot)$ are $X^{\mathrm{e}}$-continuous bilinear and linear forms, respectively; and $u\left(t^{k}\right)$ denotes the (here, single) control input at time $t=t^{k}$.

We next introduce a reference finite element approximation space $Y \subset Y^{\mathrm{e}}\left(\subset X^{\mathrm{e}}\right)$ of very large dimension $\mathcal{N}$; we further define $X \equiv X^{\mathrm{e}}$. Note that $Y$ and $X$ shall inherit the inner product and norm from $Y^{\mathrm{e}}$ and $X^{\mathrm{e}}$, respectively. Our reference (or "truth") finite element approximation $y\left(\mu, t^{k}\right) \in Y$ to the semi-discrete problem (2) is then given by

$$
m\left(y\left(\mu, t^{k}\right), v ; \mu\right)+\Delta t a\left(y\left(\mu, t^{k}\right), v ; \mu\right)=m\left(y\left(\mu, t^{k-1}\right), v ; \mu\right)+\Delta t b(v ; \mu) u\left(t^{k}\right), \quad \forall v \in Y, \forall k \in \mathbb{K},
$$

with initial condition $y\left(\mu, t^{0}\right)=0$; we then evaluate the output $s\left(\mu, t^{k}\right) \in \boldsymbol{R}$ from

$$
s\left(\mu, t^{k}\right)=\ell\left(y\left(\mu, t^{k}\right)\right), \quad \forall k \in \mathbb{K} .
$$

We shall assume - hence the appellation "truth" - that the discretization is sufficiently rich such that $y\left(\mu, t^{k}\right)$ and $y^{\mathrm{e}}\left(\mu, t^{k}\right)$ and hence $s\left(\mu, t^{k}\right)$ and $s^{\mathrm{e}}\left(\mu, t^{k}\right)$ are indistinguishable. The reduced-basis approximation shall be built upon our reference finite element approximation, and the reduced-basis error will thus be evaluated with respect to $y\left(\mu, t^{k}\right) \in Y$. Clearly, our methods must remain computationally efficient and stable as $\mathcal{N} \rightarrow \infty$.

We shall make the following assumptions. First, we assume that the bilinear forms $a(\cdot, \cdot ; \mu)$ and $m(\cdot, \cdot ; \mu)$ are continuous,

$$
\begin{aligned}
a(w, v ; \mu) & \leq \gamma(\mu)\|w\|_{Y}\|v\|_{Y} \leq \gamma_{0}\|w\|_{Y}\|v\|_{Y}, \quad \forall w, v \in Y, \quad \forall \mu \in \mathcal{D}, \\
m(w, v ; \mu) & \leq \rho(\mu)\|w\|_{X}\|v\|_{X} \leq \rho_{0}\|w\|_{X}\|v\|_{X}, \quad \forall w, v \in Y, \quad \forall \mu \in \mathcal{D} ;
\end{aligned}
$$

coercive,

$$
\begin{array}{ll}
0<\alpha_{0} \leq \alpha(\mu) \equiv \inf _{v \in Y} \frac{a(v, v ; \mu)}{\|v\|_{Y}^{2}}, \quad \forall \mu \in \mathcal{D}, \\
0<\sigma_{0} \leq \sigma(\mu) \equiv \inf _{v \in Y} \frac{m(v, v ; \mu)}{\|v\|_{X}^{2}}, \quad \forall \mu \in \mathcal{D} ;
\end{array}
$$

and symmetric, $a(v, w ; \mu)=a(w, v ; \mu), \forall w, v \in Y, \forall \mu \in \mathcal{D}$, and $m(v, w ; \mu)=m(w, v ; \mu), \forall w, v \in X, \forall \mu \in \mathcal{D}$. (We (plausibly) suppose that $\gamma_{0}, \rho_{0}, \alpha_{0}$, and $\sigma_{0}$ may be chosen independent of $\mathcal{N}$.) We also require that the linear forms $b(\cdot ; \mu): Y \rightarrow \boldsymbol{R}$ and $\ell(\cdot): Y \rightarrow \boldsymbol{R}$ be bounded with respect to $\|\cdot\|_{Y}$ and $\|\cdot\|_{X}$, respectively.

Second, we shall assume that $a, m$, and $b$ depend affinely on the parameter $\mu$ and can be expressed as

$$
\begin{aligned}
a(w, v ; \mu) & =\sum_{q=1}^{Q_{a}} \Theta_{a}^{q}(\mu) a^{q}(w, v), \quad \forall w, v \in Y, \forall \mu \in \mathcal{D}, \\
m(w, v ; \mu) & =\sum_{q=1}^{Q_{m}} \Theta_{m}^{q}(\mu) m^{q}(w, v), \quad \forall w, v \in Y, \forall \mu \in \mathcal{D}, \\
b(v ; \mu) & =\sum_{q=1}^{Q_{b}} \Theta_{b}^{q}(\mu) b^{q}(v), \quad \forall v \in Y, \forall \mu \in \mathcal{D},
\end{aligned}
$$

for some (preferably) small integers $Q_{a, m, b}$. Here, the functions $\Theta_{a, m, b}^{q}(\mu): \mathcal{D} \rightarrow \mathbb{R}$ depend on $\mu$, but the continuous forms $a^{q}, m^{q}$, and $b^{q}$ do not depend on $\mu$. This affine parameter dependence is crucial for the 
computational efficiency of the proposed method; however, see $[6,41]$ for extensions to the non-affine and nonlinear case. For simplicity of exposition, we assume that the linear form $\ell$ does not depend on the parameter; however, (affine) parameter dependence is readily admitted.

Third, and finally, we require that all linear and bilinear forms are independent of time - the system is thus linear time-invariant (LTI). This is true for many physical problems governed by parabolic PDEs, with the most notable exception of deforming domains. We point out that an important application which often satisfies all of our assumptions is the classical heat equation [35]; we shall provide a detailed example in Section 6.

To ensure rapid convergence of the reduced-basis output approximation we introduce a dual (or adjoint) problem which shall evolve backward in time [8]. Invoking the LTI property we can express the adjoint for the output at time $t^{L}, 1 \leq L \leq K$, as $\psi_{L}\left(\mu, t^{k}\right)=\Psi\left(\mu, t^{K-L+k}\right), 1 \leq k \leq L$, where $\Psi\left(\mu, t^{k}\right) \in Y$ satisfies

$$
m\left(v, \Psi\left(\mu, t^{k}\right) ; \mu\right)+\Delta t a\left(v, \Psi\left(\mu, t^{k}\right) ; \mu\right)=m\left(v, \Psi\left(\mu, t^{k+1}\right) ; \mu\right), \quad \forall v \in Y, \forall k \in \mathbb{K},
$$

with final condition

$$
m\left(v, \Psi\left(\mu, t^{K+1}\right) ; \mu\right) \equiv \ell(v), \quad \forall v \in Y .
$$

Thus, to obtain $\psi_{L}\left(\mu, t^{k}\right), 1 \leq k \leq L, \forall L \in \mathbb{K}$, we solve once for $\Psi\left(\mu, t^{k}\right), \forall k \in \mathbb{K}$, and then appropriately shift the result - we do not need to solve $K$ separate dual problems. (Note the issue of "rough" final conditions output functionals - is implicitly addressed in our temporal discretization and truth approximation.)

The method presented here easily extends to nonzero initial conditions with affine parameter dependence, to multiple control inputs and outputs, and also to nonsymmetric problems such as the convection-diffusion equation. We also note that, given a specific input $u\left(t^{k}\right), \forall k \in \mathbb{K}$, our results directly carry over to the linear time-varying (LTV) case; we can no longer, however, invoke the shift property of the dual problem - which renders the calculation of our output bound more cumbersome.

\subsection{Impulse response}

The reduced-basis subspace shall be developed as the span of solutions $y\left(\mu, t^{k}\right)$ of our "truth" approximation (3) at selected points in parameter-time space. In many cases, however, the input $u\left(t^{k}\right)$ will not be known in advance and thus we cannot solve for $y\left(\mu, t^{k}\right)$ - one such example is the optimal control problem described in the Introduction. In such situations, fortunately, we may appeal to the LTI hypothesis to justify an impulse approach, as we now describe.

We first note that the solution of any LTI system can be written as the convolution of the impulse response with the control input (Duhamel's Principle): for any control input $u\left(t^{k}\right), \forall k \in \mathbb{K}$, we can obtain $y\left(\mu, t^{k}\right)$, $\forall k \in \mathbb{K}$, from

$$
y\left(\mu, t^{k}\right)=\sum_{j=1}^{k} g\left(\mu, t^{k-j+1}\right) u\left(t^{j}\right), \quad \forall k \in \mathbb{K},
$$

where the impulse response, $g\left(\mu, t^{k}\right)$, is the solution of (3) for a unit impulse control input $u\left(t^{k}\right)=\delta_{1 k}, \forall k \in \mathbb{K}$. Equation (14) simply states that $y\left(\mu, t^{k}\right)$ is a linear combination of the impulse response $g\left(\mu, t^{j}\right), 1 \leq j \leq k$; it is thus sufficient that the reduced-basis subspace approximates well the (parameter-dependent) impulse response. It still remains to select which basis functions to retain, i.e., to determine the "best" sampling points in parameter-time space for the basis; we will address this issue in Section 5.

\section{REDUCED-BASIS METHOD}

\subsection{Approximation}

We first introduce the nested sample sets $S_{N_{\mathrm{pr}}}^{\mathrm{pr}}=\left\{\tilde{\mu}_{1}^{\mathrm{pr}} \in \tilde{\mathcal{D}}, \ldots, \tilde{\mu}_{N_{\mathrm{pr}}}^{\mathrm{pr}} \in \tilde{\mathcal{D}}\right\}, 1 \leq N_{\mathrm{pr}} \leq N_{\mathrm{pr}, \max }$, and $S_{N_{\mathrm{du}}}^{\mathrm{du}}=\left\{\tilde{\mu}_{1}^{\mathrm{du}} \in \tilde{\mathcal{D}}, \ldots, \tilde{\mu}_{N_{\mathrm{du}}}^{\mathrm{du}} \in \tilde{\mathcal{D}}\right\}, 1 \leq N_{\mathrm{du}} \leq N_{\mathrm{du}, \max }$, where $\tilde{\mu} \equiv\left(\mu, t^{k}\right)$ and $\tilde{\mathcal{D}} \equiv \mathcal{D} \times \mathbb{I} ;$ note that the samples must reside in the parameter-time space, $\tilde{\mathcal{D}}$. Here, $N_{\mathrm{pr}}$ and $N_{\mathrm{du}}$ are the dimensions of the reduced basis space 
for the primal and dual variables, respectively; in general, $S_{N_{\mathrm{pr}}}^{\mathrm{pr}} \neq S_{N_{\mathrm{du}}}^{\mathrm{du}}$ and in fact $N_{\mathrm{pr}} \neq N_{\mathrm{du}}$. We then define the associated nested Lagrangian [32] reduced-basis spaces

$$
W_{N_{\mathrm{pr}}}^{\mathrm{pr}}=\operatorname{span}\left\{\zeta_{n}^{\mathrm{pr}} \equiv y\left(\tilde{\mu}_{n}^{\mathrm{pr}}\right), 1 \leq n \leq N_{\mathrm{pr}}\right\}, \quad 1 \leq N_{\mathrm{pr}} \leq N_{\mathrm{pr}, \max }
$$

and

$$
W_{N_{\mathrm{du}}}^{\mathrm{du}}=\operatorname{span}\left\{\zeta_{n}^{\mathrm{du}} \equiv \Psi\left(\tilde{\mu}_{n}^{\mathrm{du}}\right), 1 \leq n \leq N_{\mathrm{du}}\right\}, \quad 1 \leq N_{\mathrm{du}} \leq N_{\mathrm{du}, \max },
$$

where $y\left(\tilde{\mu}_{n}^{\mathrm{pr}}\right)$ is the solution of (3) at time $t=t_{n}^{k_{n}^{\mathrm{pr}}}$ for $\mu=\mu_{n}^{\mathrm{pr}}$ and $\Psi\left(\tilde{\mu}_{n}^{\mathrm{du}}\right)$ is the solution of $(12)$ at time $t=t^{k_{n}^{\mathrm{du}}}$ for $\mu=\mu_{n}^{\mathrm{du}}$.

Our reduced-basis approximation $y_{N}\left(\mu, t^{k}\right)$ to $y\left(\mu, t^{k}\right)$ is then obtained by a standard Galerkin projection: given $\mu \in \mathcal{D}, y_{N}\left(\mu, t^{k}\right) \in W_{N_{\mathrm{pr}}}^{\mathrm{pr}}$ satisfies

$$
m\left(y_{N}\left(\mu, t^{k}\right), v ; \mu\right)+\Delta t a\left(y_{N}\left(\mu, t^{k}\right), v ; \mu\right)=m\left(y_{N}\left(\mu, t^{k-1}\right), v ; \mu\right)+\Delta t b(v ; \mu) u\left(t^{k}\right), \quad \forall v \in W_{N_{\mathrm{pr}}}^{\mathrm{pr}}, \forall k \in \mathbb{K},
$$

with initial condition $y_{N}\left(\mu, t^{0}\right)=0$. Similarly, we obtain the reduced-basis approximation $\Psi_{N}\left(\mu, t^{k}\right) \in W_{N_{\mathrm{du}}}^{\mathrm{du}}$ to $\Psi\left(\mu, t^{k}\right)$ as the solution of

$$
m\left(v, \Psi_{N}\left(\mu, t^{k}\right) ; \mu\right)+\Delta t a\left(v, \Psi_{N}\left(\mu, t^{k}\right) ; \mu\right)=m\left(v, \Psi_{N}\left(\mu, t^{k+1}\right) ; \mu\right), \quad \forall v \in W_{N_{\mathrm{du}}}^{\mathrm{du}}, \forall k \in \mathbb{K},
$$

with final condition

$$
m\left(v, \Psi_{N}\left(\mu, t^{K+1}\right) ; \mu\right) \equiv \ell(v), \quad \forall v \in W_{N_{\mathrm{du}}}^{\mathrm{du}} .
$$

Finally, we evaluate the output estimate, $s_{N}\left(\mu, t^{k}\right)$, from

$$
s_{N}\left(\mu, t^{k}\right) \equiv \ell\left(y_{N}\left(\mu, t^{k}\right)\right)+\sum_{k^{\prime}=1}^{k} R^{\mathrm{pr}}\left(\Psi_{N}\left(\mu, t^{K-k+k^{\prime}}\right) ; \mu, t^{k^{\prime}}\right) \Delta t, \quad \forall k \in \mathbb{K},
$$

where

$$
R^{\mathrm{pr}}\left(v ; \mu, t^{k}\right) \equiv b(v ; \mu) u\left(t^{k}\right)-a\left(y_{N}\left(\mu, t^{k}\right), v ; \mu\right)-\frac{1}{\Delta t} m\left(y_{N}\left(\mu, t^{k}\right)-y_{N}\left(\mu, t^{k-1}\right), v ; \mu\right), \quad \forall v \in Y, \forall k \in \mathbb{K},
$$

is the primal residual. Note that here $N \equiv\left(N_{\mathrm{pr}}, N_{\mathrm{du}}\right)$.

The critical observation is that the field variable $y\left(\mu, t^{k}\right), \forall k \in \mathbb{K}$, is not, in fact, some arbitrary member of the very high dimensional finite element space $Y$; rather, it resides, or "evolves," on a much lower dimensional manifold - in effect, a $P+1$ dimensional manifold - induced by the parametric and temporal dependence. Thus, by restricting our attention to this manifold, we can adequately approximate the field variable by a space of dimension $N_{\mathrm{pr}}, N_{\mathrm{du}} \ll \mathcal{N}$. ${ }^{1}$ This observation is fundamental to our approach, and is the basis of our approximation; we confirm the rapid convergence in Section 6.

\subsection{Computational procedure}

In this section we develop offline-online computational procedures in order to fully exploit the dimension reduction of the problem $[3,17,22,34]$. We first express $y_{N}\left(\mu, t^{k}\right)$ and $\Psi_{N}\left(\mu, t^{k}\right)$ as

$$
y_{N}\left(\mu, t^{k}\right)=\sum_{n=1}^{N_{\mathrm{pr}}} y_{N n}\left(\mu, t^{k}\right) \zeta_{n}^{\mathrm{pr}}
$$

\footnotetext{
1 In general, the field variable will be smooth in $\mu$. This may be deduced from the equations for the sensitivity derivatives; the stability and continuity properties of the partial differential operator are crucial. Note, however, that the proposed method does not require great regularity of the field variable in $x$; hence non-smooth domains (sharp corners) pose no impediment to rapid convergence.
} 
and

$$
\Psi_{N}\left(\mu, t^{k}\right)=\sum_{n=1}^{N_{\mathrm{du}}} \Psi_{N n}\left(\mu, t^{k}\right) \zeta_{n}^{\mathrm{du}},
$$

respectively. We then choose as test functions $v=\zeta_{n}^{\mathrm{pr}}, 1 \leq n \leq N_{\mathrm{pr}}$, for the primal problem (17) and $v=\zeta_{n}^{\mathrm{du}}, 1 \leq n \leq N_{\mathrm{du}}$, for the dual problem (18). (We prefer Galerkin over Petrov-Galerkin for purposes of stability.)

It then follows from (17) that $\underline{y}_{N}\left(\mu, t^{k}\right)=\left[y_{N 1}\left(\mu, t^{k}\right) y_{N}\left(\mu, t^{k}\right) \ldots y_{N} N_{\mathrm{pr}}\left(\mu, t^{k}\right)\right]^{T} \in \boldsymbol{R}^{N_{\mathrm{pr}}}$ satisfies

$$
\left(M_{N}^{\mathrm{pr}}(\mu)+\Delta t A_{N}^{\mathrm{pr}}(\mu)\right) \underline{y}_{N}\left(\mu, t^{k}\right)=M_{N}^{\mathrm{pr}}(\mu) \underline{y}_{N}\left(\mu, t^{k-1}\right)+\Delta t B_{N}^{\mathrm{pr}}(\mu) u\left(t^{k}\right), \quad \forall k \in \mathbb{K},
$$

with initial condition $y_{N n}\left(\mu, t^{0}\right)=0,1 \leq n \leq N_{\mathrm{pr}}$. Here, $M_{N}^{\mathrm{pr}}(\mu) \in \boldsymbol{R}^{N_{\mathrm{pr}} \times N_{\mathrm{pr}}}$ and $A_{N}^{\mathrm{pr}}(\mu) \in \boldsymbol{R}^{N_{\mathrm{pr}} \times N_{\mathrm{pr}}}$ are SPD matrices with entries $M_{N i, j}^{\mathrm{pr}}(\mu)=m\left(\zeta_{i}^{\mathrm{pr}}, \zeta_{j}^{\mathrm{pr}} ; \mu\right), 1 \leq i, j \leq N_{\mathrm{pr}}$, and $A_{N i, j}^{\mathrm{pr}}(\mu)=a\left(\zeta_{i}^{\mathrm{pr}}, \zeta_{j}^{\mathrm{pr}} ; \mu\right), 1 \leq i, j \leq N_{\mathrm{pr}}$, respectively; and $B_{N}^{\mathrm{pr}}(\mu) \in \boldsymbol{R}^{N_{\mathrm{pr}}}$ is the control vector with entries $B_{N i}^{\mathrm{pr}}(\mu)=b\left(\zeta_{i}^{\mathrm{pr}} ; \mu\right), 1 \leq i \leq N_{\mathrm{pr}}$.

Invoking the affine decomposition (9)-(11) we obtain

$$
\begin{aligned}
& M_{N i, j}^{\mathrm{pr}}(\mu)=m\left(\zeta_{i}^{\mathrm{pr}}, \zeta_{j}^{\mathrm{pr}} ; \mu\right)=\sum_{q=1}^{Q_{m}} \Theta_{m}^{q}(\mu) m^{q}\left(\zeta_{i}^{\mathrm{pr}}, \zeta_{j}^{\mathrm{pr}}\right), \\
& A_{N i, j}^{\mathrm{pr}}(\mu)=a\left(\zeta_{i}^{\mathrm{pr}}, \zeta_{j}^{\mathrm{pr}} ; \mu\right)=\sum_{q=1}^{Q_{a}} \Theta_{a}^{q}(\mu) a^{q}\left(\zeta_{i}^{\mathrm{pr}}, \zeta_{j}^{\mathrm{pr}}\right), \\
& B_{N i}^{\mathrm{pr}}(\mu)=b^{q}\left(\zeta_{i}^{\mathrm{pr}} ; \mu\right)=\sum_{q=1}^{Q_{b}} \Theta_{b}^{q}(\mu) b^{q}\left(\zeta_{i}^{\mathrm{pr}}\right),
\end{aligned}
$$

which can be written as

$$
M_{N}^{\mathrm{pr}}(\mu)=\sum_{q=1}^{Q_{m}} \Theta_{m}^{q}(\mu) M_{N}^{\mathrm{pr} q}, \quad A_{N}^{\mathrm{pr}}(\mu)=\sum_{q=1}^{Q_{a}} \Theta_{a}^{q}(\mu) A_{N}^{\mathrm{pr} q}, \quad B_{N}^{\mathrm{pr}}(\mu)=\sum_{q=1}^{Q_{b}} \Theta_{b}^{q}(\mu) B_{N}^{\mathrm{pr} q},
$$

where the parameter independent quantities $M_{N}^{\mathrm{pr} q} \in \boldsymbol{R}^{N_{\mathrm{pr}} \times N_{\mathrm{pr}}}, A_{N}^{\mathrm{pr} q} \in \boldsymbol{R}^{N_{\mathrm{pr}} \times N_{\mathrm{pr}}}$, and $B_{N}^{\mathrm{pr} q} \in \boldsymbol{R}^{N_{\mathrm{pr}}}$ are given by

respectively.

$$
\begin{aligned}
M_{N i, j}^{\mathrm{pr} q} & =m^{q}\left(\zeta_{i}^{\mathrm{pr}}, \zeta_{j}^{\mathrm{pr}}\right), 1 \leq i, j \leq N_{\mathrm{pr}, \max }, 1 \leq q \leq Q_{m}, \\
A_{N i, j}^{\mathrm{pr} q} & =a^{q}\left(\zeta_{i}^{\mathrm{pr}}, \zeta_{j}^{\mathrm{pr}}\right), \quad 1 \leq i, j \leq N_{\mathrm{pr}, \max }, 1 \leq q \leq Q_{a}, \\
B_{N i}^{\mathrm{pr} q} & =b^{q}\left(\zeta_{i}^{\mathrm{pr}}\right), \quad 1 \leq i \leq N_{\mathrm{pr}, \max }, 1 \leq q \leq Q_{b},
\end{aligned}
$$

A similar computational procedure for the dual problem (18)-(19) and the residual correction term in (20) can also be developed. The details of this derivation and the definitions of the necessary quantities are summarized in Appendix A.1.

The offline-online decomposition is now clear. In the offline stage - performed only once - we first solve for the $\zeta_{n}^{\mathrm{pr}}, 1 \leq n \leq N_{\mathrm{pr}, \max }$ and $\zeta_{n}^{\mathrm{du}}, 1 \leq n \leq N_{\mathrm{du} \text {,max }}$; we then compute and store the $\mu$-independent quantities in (29) for the primal problem, (91) for the dual problem, and (94) for the output estimate. The computational cost is therefore $O\left(K\left(N_{\mathrm{pr}, \max }+N_{\mathrm{du}, \max }\right)\right)$ solutions of the underlying $\mathcal{N}$-dimensional "truth" finite element approximation and $O\left(\left(N_{\mathrm{pr}, \max }^{2}+N_{\mathrm{du}, \max }^{2}+N_{\mathrm{pr}, \max } N_{\mathrm{du}, \max }\right)\left(Q_{a}+Q_{m}\right)\right) \mathcal{N}$-inner products; the storage requirements are also $O\left(\left(N_{\mathrm{pr}, \max }^{2}+N_{\mathrm{du}, \max }^{2}+N_{\mathrm{pr}, \max } N_{\mathrm{du}, \max }\right)\left(Q_{a}+Q_{m}\right)\right)$.

In the online stage - performed many times, for each new parameter value $\mu$ - we first assemble the reducedbasis matrices (28), (90), and (93); this requires $O\left(\left(N_{\mathrm{pr}}^{2}+N_{\mathrm{du}}^{2}+N_{\mathrm{pr}} N_{\mathrm{du}}\right)\left(Q_{a}+Q_{m}\right)\right)$ operations. We then solve the primal and dual problem for $\underline{y}_{N}\left(\mu, t^{k}\right)$ and $\underline{\Psi}_{N}\left(\mu, t^{k}\right)$, respectively; since the reduced-basis matrices 
are in general full, the operation count (based on LU factorization and our LTI assumption) is $O\left(N_{\mathrm{pr}}^{3}+N_{\mathrm{du}}^{3}+\right.$ $\left.K\left(N_{\mathrm{pr}}^{2}+N_{\mathrm{du}}^{2}\right)\right)$. Finally, given $\underline{y}_{N}\left(\mu, t^{k}\right)$ and $\underline{\Psi}_{N}\left(\mu, t^{k}\right)$ we evaluate the output estimate $s_{N}\left(\mu, t^{k}\right)$ from $(92)$ at a cost of $O\left(2 k N_{\mathrm{pr}} N_{\mathrm{du}}\right)$; note that the calculation of all outputs $s_{N}\left(\mu, t^{k}\right), \forall k \in \mathbb{K}$, requires $O\left(K(K+1) N_{\mathrm{pr}} N_{\mathrm{du}}\right)$ operations.

Thus, as required in the many-query or real-time contexts, the online complexity is independent of $\mathcal{N}$, the dimension of the underlying "truth" finite element approximation space. Since $N_{\text {pr }}, N_{\text {du }} \ll \mathcal{N}$ we expect significant computational savings in the online stage relative to classical discretization and solution approaches.

Finally, we note that classical model-order reduction techniques, such as modal decomposition [12] and POD [2], require the evaluation of a new set of eigenmodes or basis functions - and thus a return to the (very fine) "truth" approximation - for each new parameter value encountered. In contrast, reduced-basis methods do not need to return to the "truth" approximation in the online stage, and are therefore far more efficient in evaluating input-output relationships for many different parameter values.

\section{A POSTERIORI ERROR ESTIMATION}

From Section 3 we know that we can efficiently obtain the output estimate, $s_{N}\left(\mu, t^{k}\right)$, for the output of interest, $s\left(\mu, t^{k}\right)$ : the online complexity depends only on $N_{\mathrm{pr}}$ and $N_{\mathrm{du}}$, the dimensions of the reduced-basis spaces for the primal and dual variable, respectively. However, we do not yet know if $s_{N}\left(\mu, t^{k}\right)$ is indeed a good approximation to $s\left(\mu, t^{k}\right)$, i.e., is $\left|s\left(\mu, t^{k}\right)-s_{N}\left(\mu, t^{k}\right)\right| \leq \epsilon_{\mathrm{tol}}^{s}$, where $\epsilon_{\mathrm{tol}}^{s}$ is a maximum acceptable error? Or conversely, is our approximation "too good," i.e., is $\left|s\left(\mu, t^{k}\right)-s_{N}\left(\mu, t^{k}\right)\right| \ll \epsilon_{\text {tol }}^{s}$ - that is, is $N_{\text {pr }}$ or $N_{\text {du }}$ too large, with associated detriment to the online efficiency? It should also be evident that the approximation properties do not only depend on the size of $N_{\mathrm{pr}}$ and $N_{\mathrm{du}}$, but also on the choice of the sampling sets $S_{N_{\mathrm{pr}}}^{\mathrm{pr}}$ and $S_{N_{\mathrm{du}}}^{\mathrm{du}}$ and associated reduced-basis spaces $W_{N_{\mathrm{pr}}}^{\mathrm{pr}}$ and $W_{N_{\mathrm{du}}}^{\mathrm{du}}$.

We thus need to develop rigorous a posteriori error estimators which will help us to $(i)$ assess the error introduced by our reduced-basis approximation (relative to the "truth" finite element approximation); and (ii) devise an "optimal" and efficient procedure for selecting the sample sets $S_{N_{\mathrm{pr}}}^{\mathrm{pr}}$ and $S_{N_{\mathrm{du}}}^{\mathrm{du}}$. Surprisingly, a posteriori error estimation for reduced-basis approximations has received very little attention in the past. A family of rigorous error estimators for reduced-basis approximations of a wide class of elliptic PDEs is introduced in $[22,34,41-43]$; we will now extend these ideas to time-dependent (parabolic) partial differential equations. Our approach here is a simplification and generalization of earlier efforts in this direction [39]. ${ }^{2}$

We remark that the development of the error bounds presented below is not limited to the reduced-basis approximation described in this paper: with suitable hypotheses, we may consider "any" stable ODE or PDE system and associated reduced-order model.

\subsection{Error bounds}

\subsubsection{Preliminaries}

To begin, we assume that we are given positive lower bounds for the coercivity constants, $\alpha(\mu)$ and $\sigma(\mu)$ : $\hat{\alpha}(\mu): \mathcal{D} \rightarrow \boldsymbol{R}_{+}$satisfies

and $\hat{\sigma}(\mu): \mathcal{D} \rightarrow \boldsymbol{R}_{+}$satisfies

$$
\alpha(\mu) \geq \hat{\alpha}(\mu) \geq \hat{\alpha}_{0}>0, \quad \forall \mu \in \mathcal{D}
$$

$$
\sigma(\mu) \geq \hat{\sigma}(\mu) \geq \hat{\sigma}_{0}>0, \quad \forall \mu \in \mathcal{D}
$$

2 Concurrently with our submission, Rovas, Machiels, and Maday submitted a manuscript [38] summarizing the earlier work [39]. Although our paper here and [38] address common issues, the two approaches differ significantly in the class of problems treated (known [38] vs. unknown temporal forcing/controls here), in the output families considered (scalars [38] vs. functions of (discrete) time here), in the temporal treatment (continuous in time or arbitrary-order Discontinuous-Galerkin discretization [38] vs. loworder finite-difference here), in the sampling procedures (log-random [38] vs. greedy [9] here), and also in the emphasis on a priori convergence results (rigorous [38] vs. empirical here). As a result, the a posteriori error estimators and associated computational procedures (e.g., for the necessary adjoints) are quite different, as are the families of possible applications. 
various recipes for this construction can be found in [34,44]. We next introduce the dual norm of the primal residual

$$
\varepsilon_{N_{\mathrm{pr}}}^{\mathrm{pr}}\left(\mu, t^{k}\right) \equiv \sup _{v \in Y} \frac{R^{\mathrm{pr}}\left(v ; \mu, t^{k}\right)}{\|v\|_{Y}}, \quad \forall k \in \mathbb{K}
$$

and the dual norm of the dual residual

$$
\varepsilon_{N_{\mathrm{du}}}^{\mathrm{du}}\left(\mu, t^{k}\right) \equiv \sup _{v \in Y} \frac{R^{\mathrm{du}}\left(v ; \mu, t^{k}\right)}{\|v\|_{Y}}, \quad \forall k \in \mathbb{K},
$$

where

$$
R^{\mathrm{du}}\left(v ; \mu, t^{k}\right) \equiv-a\left(v, \Psi_{N}\left(\mu, t^{k}\right) ; \mu\right)-\frac{1}{\Delta t} m\left(v, \Psi_{N}\left(\mu, t^{k}\right)-\Psi_{N}^{k+1}(\mu) ; \mu\right), \quad \forall v \in Y, \forall k \in \mathbb{K},
$$

is the dual residual. We also specify the inner products

$$
(v, w)_{Y} \equiv a\left(v, w ; \mu_{\mathrm{ref}(\mathrm{s})}\right), \quad \forall v, w \in Y,
$$

and

$$
(v, w)_{X} \equiv m\left(v, w ; \mu_{\operatorname{ref}(\mathrm{s})}\right), \quad \forall v, w \in Y,
$$

for some constant reference value(s) $\mu_{\operatorname{ref}(\mathrm{s})}$, and recall that $\|\cdot\|_{Y}=(\cdot, \cdot)_{Y}^{1 / 2},\|\cdot\|_{X}=(\cdot, \cdot)_{X}^{1 / 2}$.

We now present and prove the bounding properties for the errors in the primal variable, the dual variable, and the output estimate. Throughout this section we assume that the "truth" solutions $y\left(\mu, t^{k}\right)$ and $\Psi\left(\mu, t^{k}\right)$ satisfy (3) and (12), respectively, and the corresponding reduced-basis approximations $y_{N}\left(\mu, t^{k}\right)$ and $\Psi_{N}\left(\mu, t^{k}\right)$ satisfy (17) and (18), respectively. We emphasize that our error bounds are very classical, based entirely on standard stability results invoked in a priori analyses [35]; the critical new ingredient - tailored to the reducedbasis context - is the offline-online computational procedure of Section 4.2.

\subsubsection{Primal variable}

We obtain the following result for the error in the primal variable.

Proposition 4.1. Let $e^{\operatorname{pr}}\left(\mu, t^{k}\right) \equiv y\left(\mu, t^{k}\right)-y_{N}\left(\mu, t^{k}\right)$ be the error in the primal variable and define the "spatiotemporal" energy norm

$$
\left\|\mid v\left(\mu, t^{k}\right)\right\|^{p^{\mathrm{pr}}} \equiv\left(m\left(v\left(\mu, t^{k}\right), v\left(\mu, t^{k}\right) ; \mu\right)+\sum_{k^{\prime}=1}^{k} a\left(v\left(\mu, t^{k^{\prime}}\right), v\left(\mu, t^{k^{\prime}}\right) ; \mu\right) \Delta t\right)^{\frac{1}{2}}, \quad \forall v \in Y .
$$

The error in the primal variable is then bounded by

$$
\left\|\left|e^{\mathrm{pr}}\left(\mu, t^{k}\right)\right|\right\|^{\mathrm{pr}} \leq \Delta_{N_{\mathrm{pr}}}^{\mathrm{pr}}\left(\mu, t^{k}\right), \quad \forall \mu \in \mathcal{D}, \forall k \in \mathbb{K},
$$

where the error bound $\Delta_{N_{\mathrm{pr}}}^{\mathrm{pr}}\left(\mu, t^{k}\right)$ is defined as

$$
\Delta_{N_{\mathrm{pr}}}^{\mathrm{pr}}\left(\mu, t^{k}\right) \equiv\left(\frac{\Delta t}{\hat{\alpha}(\mu)} \sum_{k^{\prime}=1}^{k} \varepsilon_{N_{\mathrm{pr}}}^{\mathrm{pr}}\left(\mu, t^{k^{\prime}}\right)^{2}\right)^{\frac{1}{2}}
$$

and $\varepsilon_{N_{\mathrm{pr}}}^{\mathrm{pr}}\left(\mu, t^{k}\right)$ is the dual norm of the primal residual defined in (32).

Proof. We immediately derive from (3) and (21) that $e^{\mathrm{pr}}\left(\mu, t^{k}\right)=y\left(\mu, t^{k}\right)-y_{N}\left(\mu, t^{k}\right)$ satisfies

$$
m\left(e^{\mathrm{pr}}\left(\mu, t^{k}\right), v ; \mu\right)+\Delta t a\left(e^{\mathrm{pr}}\left(\mu, t^{k}\right), v ; \mu\right)=m\left(e^{\mathrm{pr}}\left(\mu, t^{k-1}\right), v ; \mu\right)+\Delta t R^{\mathrm{pr}}\left(v ; \mu, t^{k}\right), \quad \forall v \in Y, \forall k \in \mathbb{K},
$$


where $e^{\text {pr }}\left(\mu, t^{0}\right)=0$ since $y\left(\mu, t^{0}\right)=y_{N}\left(\mu, t^{0}\right)=0$ by assumption. We now choose $v=e^{\text {pr }}\left(\mu, t^{k}\right)$, invoke the Cauchy-Schwarz inequality for the cross term $m\left(e^{\mathrm{pr}}\left(\mu, t^{k-1}\right), e^{\mathrm{pr}}\left(\mu, t^{k}\right) ; \mu\right)$, and apply (32) to obtain

$$
\begin{aligned}
& m\left(e^{\mathrm{pr}}\left(\mu, t^{k}\right), e^{\mathrm{pr}}\left(\mu, t^{k}\right) ; \mu\right)+\Delta t a\left(e^{\mathrm{pr}}\left(\mu, t^{k}\right), e^{\mathrm{pr}}\left(\mu, t^{k}\right) ; \mu\right) \\
& \leq m^{\frac{1}{2}}\left(e^{\mathrm{pr}}\left(\mu, t^{k}\right), e^{\mathrm{pr}}\left(\mu, t^{k}\right) ; \mu\right) m^{\frac{1}{2}}\left(e^{\mathrm{pr}}\left(\mu, t^{k-1}\right), e^{\mathrm{pr}}\left(\mu, t^{k-1}\right) ; \mu\right) \\
& +\Delta t \varepsilon_{N_{\mathrm{pr}}}^{\mathrm{pr}}\left(\mu, t^{k}\right)\left\|e^{\mathrm{pr}}\left(\mu, t^{k}\right)\right\|_{Y}, \quad \forall k \in \mathbb{K} .
\end{aligned}
$$

We now recall the identity (for $c \in \mathbb{R}, d \in \mathbb{R}, \rho \in \mathbb{R}_{+}$)

$$
2|c||d| \leq \frac{1}{\rho^{2}} c^{2}+\rho^{2} d^{2}
$$

which we apply twice: first, choosing $c=m^{\frac{1}{2}}\left(e^{\mathrm{pr}}\left(\mu, t^{k}\right), e^{\mathrm{pr}}\left(\mu, t^{k}\right) ; \mu\right), d=m^{\frac{1}{2}}\left(e^{\mathrm{pr}}\left(\mu, t^{k-1}\right), e^{\mathrm{pr}}\left(\mu, t^{k-1}\right) ; \mu\right)$, and $\rho=1$, we obtain

$$
\begin{aligned}
2 m^{\frac{1}{2}}\left(e^{\mathrm{pr}}\left(\mu, t^{k}\right), e^{\mathrm{pr}}\left(\mu, t^{k}\right) ; \mu\right) m^{\frac{1}{2}}\left(e^{\mathrm{pr}}\left(\mu, t^{k-1}\right), e^{\mathrm{pr}}\left(\mu, t^{k-1}\right) ; \mu\right) \\
\quad \leq m\left(e^{\mathrm{pr}}\left(\mu, t^{k-1}\right), e^{\mathrm{pr}}\left(\mu, t^{k-1}\right) ; \mu\right)+m\left(e^{\mathrm{pr}}\left(\mu, t^{k}\right), e^{\mathrm{pr}}\left(\mu, t^{k}\right) ; \mu\right)
\end{aligned}
$$

and second, choosing $c=\varepsilon_{N_{\mathrm{pr}}}^{\mathrm{pr}}\left(\mu, t^{k}\right), d=\left\|e^{\mathrm{pr}}\left(\mu, t^{k}\right)\right\|_{Y}$, and $\rho=\hat{\alpha}(\mu)^{\frac{1}{2}}$ we have

$$
2 \varepsilon_{N_{\mathrm{pr}}}^{\mathrm{pr}}\left(\mu, t^{k}\right)\left\|e^{\mathrm{pr}}\left(\mu, t^{k}\right)\right\|_{Y} \leq \frac{1}{\hat{\alpha}(\mu)} \varepsilon_{N_{\mathrm{pr}}}^{\mathrm{pr}}\left(\mu, t^{k}\right)^{2}+\hat{\alpha}(\mu)\left\|e^{\mathrm{pr}}\left(\mu, t^{k}\right)\right\|_{Y}^{2}
$$

Combining (41), (43), and (44), and invoking (7) and (30), we obtain

$$
\begin{aligned}
m\left(e^{\mathrm{pr}}\left(\mu, t^{k}\right), e^{\mathrm{pr}}\left(\mu, t^{k}\right) ; \mu\right)-m\left(e^{\mathrm{pr}}\left(\mu, t^{k-1}\right)\right. & \left., e^{\mathrm{pr}}\left(\mu, t^{k-1}\right) ; \mu\right) \\
& +\Delta t a\left(e^{\mathrm{pr}}\left(\mu, t^{k}\right), e^{\mathrm{pr}}\left(\mu, t^{k}\right) ; \mu\right) \leq \frac{\Delta t}{\hat{\alpha}(\mu)} \varepsilon_{N_{\mathrm{pr}}}^{\mathrm{pr}}\left(\mu, t^{k}\right)^{2}, \quad \forall k \in \mathbb{K} .
\end{aligned}
$$

We now perform the sum from $k^{\prime}=1$ to $k$ and recall that $e^{\operatorname{pr}}\left(\mu, t^{0}\right)=0$, leading to

$$
m\left(e^{\mathrm{pr}}\left(\mu, t^{k}\right), e^{\mathrm{pr}}\left(\mu, t^{k}\right) ; \mu\right)+\sum_{k^{\prime}=1}^{k} \Delta t a\left(e^{\mathrm{pr}}\left(\mu, t^{k^{\prime}}\right), e^{\mathrm{pr}}\left(\mu, t^{k^{\prime}}\right) ; \mu\right) \leq \frac{\Delta t}{\hat{\alpha}(\mu)} \sum_{k^{\prime}=1}^{k} \varepsilon_{N_{\mathrm{pr}}}^{\mathrm{pr}}\left(\mu, t^{k^{\prime}}\right)^{2}, \quad \forall k \in \mathbb{K},
$$

which is the result stated in Proposition 4.1.

\subsubsection{Dual variable}

Before proceeding with the error bounds for the dual variable we have to pay special attention to the final condition of the dual problem. The primal error at time zero, $e^{\mathrm{pr}}\left(\mu, t^{0}\right)$, vanishes (for our zero initial conditions) and therefore does not contribute to the error bound. For the dual problem, however, the error at the final time $t^{K+1}, e^{\mathrm{du}}\left(\mu, t^{K+1}\right) \equiv \Psi\left(\mu, t^{K+1}\right)-\Psi_{N}\left(\mu, t^{K+1}\right)$ is - in general - nonzero since $\Psi\left(\mu, t^{K+1}\right)$ is not necessarily a member of $W_{N_{\mathrm{du}}}^{\mathrm{du}}$. Instead, we obtain from (13) that $e^{\mathrm{du}}\left(\mu, t^{K+1}\right)$ satisfies

$$
m\left(v, e^{\mathrm{du}}\left(\mu, t^{K+1}\right) ; \mu\right)=R^{\Psi_{f}}(v ; \mu), \quad \forall v \in Y
$$

where

$$
R^{\Psi_{f}}(v ; \mu) \equiv \ell(v)-m\left(v, \Psi_{N}\left(\mu, t^{K+1}\right) ; \mu\right), \quad \forall v \in Y,
$$

is the residual associated to the final condition. It can be shown that $e^{\mathrm{du}}\left(\mu, t^{K+1}\right)$ satisfies the following bound $[34,43]$. 
Lemma 4.2. The error $e^{\mathrm{du}}\left(\mu, t^{K+1}\right) \equiv \Psi\left(\mu, t^{K+1}\right)-\Psi_{N}\left(\mu, t^{K+1}\right)$ is bounded by

$$
\left\|e^{\mathrm{du}}\left(\mu, t^{K+1}\right)\right\|_{X} \leq \Delta_{N_{\mathrm{du}}}^{\Psi_{f}}(\mu) \equiv \frac{\varepsilon_{N_{\mathrm{du}}}^{\Psi_{f}}(\mu)}{\hat{\sigma}(\mu)}
$$

where

$$
\varepsilon_{N_{\mathrm{du}}}^{\Psi_{f}}(\mu) \equiv \sup _{v \in Y} \frac{R^{\Psi_{f}}(v ; \mu)}{\|v\|_{X}}
$$

is the dual norm of the residual associated to the final condition.

It directly follows from Lemma 4.2 and (47) that

$$
\begin{aligned}
m\left(e^{\mathrm{du}}\left(\mu, t^{K+1}\right), e^{\mathrm{du}}\left(\mu, t^{K+1}\right) ; \mu\right) & =R^{\Psi_{f}}\left(e^{\mathrm{du}}\left(\mu, t^{K+1}\right) ; \mu\right) \leq \varepsilon_{N_{\mathrm{du}}}^{\Psi_{f}}(\mu)\left\|e^{\mathrm{du}}\left(\mu, t^{K+1}\right)\right\|_{X} \\
& \leq \hat{\sigma}(\mu) \Delta_{N_{\mathrm{du}}}^{\Psi_{f}}(\mu)^{2} .
\end{aligned}
$$

Note that for the special case in which the bilinear form $m$ is parameter-independent, we can guarantee that $\Psi\left(\mu, t^{K+1}\right)$ is a member of $W_{N_{\mathrm{du}}}^{\mathrm{du}}$ and thus $e^{\mathrm{du}}\left(\mu, t^{K+1}\right)$ is identically zero.

We are now ready to prove the bounding property for the dual problem.

Proposition 4.3. Let $e^{\mathrm{du}}\left(\mu, t^{k}\right) \equiv \Psi\left(\mu, t^{k}\right)-\Psi_{N}\left(\mu, t^{k}\right)$ be the error in the dual variable and define

$$
\| v\left(\mu, t^{k}\right)||^{\mathrm{du}} \equiv\left(m\left(v\left(\mu, t^{k}\right), v\left(\mu, t^{k}\right) ; \mu\right)+\sum_{k^{\prime}=k}^{K} a\left(v\left(\mu, t^{k^{\prime}}\right), v\left(\mu, t^{k^{\prime}}\right) ; \mu\right) \Delta t\right)^{\frac{1}{2}} .
$$

The error in the dual variable is then bounded by

$$
\left\|e^{\mathrm{du}}\left(\mu, t^{k}\right)\right\| \|^{\mathrm{du}} \leq \Delta_{N_{\mathrm{du}}}^{\mathrm{du}}\left(\mu, t^{k}\right), \quad \forall \mu \in \mathcal{D}, \forall k \in \mathbb{K},
$$

where the error bound $\Delta_{N_{\mathrm{du}}}^{\mathrm{du}}\left(\mu, t^{k}\right)$ is defined as

$$
\Delta_{N_{\mathrm{du}}}^{\mathrm{du}}\left(\mu, t^{k}\right) \equiv\left(\frac{\Delta t}{\hat{\alpha}(\mu)} \sum_{k^{\prime}=k}^{K} \varepsilon_{N_{\mathrm{du}}}^{\mathrm{du}}\left(\mu, t^{k^{\prime}}\right)^{2}+\hat{\sigma}(\mu) \Delta_{N_{\mathrm{du}}}^{\Psi_{f}}(\mu)^{2}\right)^{\frac{1}{2}}
$$

and $\varepsilon_{N_{\mathrm{du}}}^{\mathrm{du}}\left(\mu, t^{k}\right)$ is the dual norm of the dual residual defined in (33).

Proof. We immediately derive from (12) and (34) that $e^{\mathrm{du}}\left(\mu, t^{k}\right)=\Psi\left(\mu, t^{k}\right)-\Psi_{N}\left(\mu, t^{k}\right)$ satisfies

$$
m\left(v, e^{\mathrm{du}}\left(\mu, t^{k}\right) ; \mu\right)+\Delta t a\left(v, e^{\mathrm{du}}\left(\mu, t^{k}\right) ; \mu\right)=m\left(v, e^{\mathrm{du}}\left(\mu, t^{k+1}\right) ; \mu\right)+\Delta t R^{\mathrm{du}}\left(v ; \mu, t^{k}\right), \quad \forall v \in Y, \forall k \in \mathbb{K},
$$

with final condition $m\left(v, e^{\mathrm{du}}\left(\mu, t^{K+1}\right) ; \mu\right)=R^{\Psi_{f}}(v ; \mu), \forall v \in Y$. Choosing $v=e^{\mathrm{du}}\left(\mu, t^{k}\right)$, invoking the CauchySchwarz inequality, and applying (33) we obtain

$$
\begin{aligned}
m\left(e^{\mathrm{du}}\left(\mu, t^{k}\right), e^{\mathrm{du}}\left(\mu, t^{k}\right) ; \mu\right) & +\Delta t a\left(e^{\mathrm{du}}\left(\mu, t^{k}\right), e^{\mathrm{du}}\left(\mu, t^{k}\right) ; \mu\right) \\
\leq & m^{\frac{1}{2}}\left(e^{\mathrm{du}}\left(\mu, t^{k+1}\right), e^{\mathrm{du}}\left(\mu, t^{k+1}\right) ; \mu\right) m^{\frac{1}{2}}\left(e^{\mathrm{du}}\left(\mu, t^{k}\right), e^{\mathrm{du}}\left(\mu, t^{k}\right) ; \mu\right) \\
& +\Delta t \varepsilon_{N_{\mathrm{du}}}^{\mathrm{du}}\left(\mu, t^{k}\right)\left\|e^{\mathrm{du}}\left(\mu, t^{k}\right)\right\|_{Y}, \quad \forall k \in \mathbb{K} .
\end{aligned}
$$


We now apply (42) twice: first, with $c=m^{\frac{1}{2}}\left(e^{\mathrm{du}}\left(\mu, t^{k+1}\right), e^{\mathrm{du}}\left(\mu, t^{k+1}\right) ; \mu\right), d=m^{\frac{1}{2}}\left(e^{\mathrm{du}}\left(\mu, t^{k}\right), e^{\mathrm{du}}\left(\mu, t^{k}\right) ; \mu\right)$, and $\rho=1$; and second, with $c=\varepsilon_{N_{\mathrm{du}}}^{\mathrm{du}}\left(\mu, t^{k}\right), d=\left\|e^{\mathrm{du}}\left(\mu, t^{k}\right)\right\|_{Y}$, and $\rho=\hat{\alpha}(\mu)^{\frac{1}{2}}$. Invoking $(7)$ and $(30)$, we arrive at

$$
\begin{aligned}
& m\left(e^{\mathrm{du}}\left(\mu, t^{k}\right), e^{\mathrm{du}}\left(\mu, t^{k}\right) ; \mu\right)-m\left(e^{\mathrm{du}}\left(\mu, t^{k+1}\right), e^{\mathrm{du}}\left(\mu, t^{k+1}\right) ; \mu\right) \\
& \quad+\Delta t a\left(e^{\mathrm{du}}\left(\mu, t^{k}\right), e^{\mathrm{du}}\left(\mu, t^{k}\right) ; \mu\right) \leq \frac{\Delta t}{\hat{\alpha}(\mu)} \varepsilon_{N_{\mathrm{du}}}^{\mathrm{du}}\left(\mu, t^{k}\right)^{2}, \quad \forall k \in \mathbb{K},
\end{aligned}
$$

We now perform the sum from $k^{\prime}=k$ to $K$ and invoke (51) to obtain

$$
\begin{aligned}
m\left(e^{\mathrm{du}}\left(\mu, t^{k}\right), e^{\mathrm{du}}\left(\mu, t^{k}\right) ; \mu\right)+\sum_{k^{\prime}=k}^{K} \Delta t a\left(e^{\mathrm{du}}\left(\mu, t^{k^{\prime}}\right), e^{\mathrm{du}}\left(\mu, t^{k^{\prime}}\right) ; \mu\right) & \\
& \leq \frac{\Delta t}{\hat{\alpha}(\mu)} \sum_{k^{\prime}=k}^{K} \varepsilon_{N_{\mathrm{du}}}^{\mathrm{du}}\left(\mu, t^{k^{\prime}}\right)^{2}+\hat{\sigma}(\mu) \Delta_{N_{\mathrm{du}}}^{\Psi_{f}}(\mu)^{2}, \quad \forall k \in \mathbb{K},
\end{aligned}
$$

which is the result stated in Proposition 4.3.

\subsubsection{Output bounds}

Finally, the error bound for the output estimate is given in the following proposition.

Proposition 4.4. Let the output of interest, $s\left(\mu, t^{k}\right)$, and the reduced-basis output estimate, $s_{N}\left(\mu, t^{k}\right)$, be given by

$$
s\left(\mu, t^{k}\right)=\ell\left(y\left(\mu, t^{k}\right)\right), \quad \forall \mu \in \mathcal{D}, \forall k \in \mathbb{K}
$$

and

$$
s_{N}\left(\mu, t^{k}\right)=\ell\left(y_{N}\left(\mu, t^{k}\right)\right)+\sum_{k^{\prime}=1}^{k} R^{\mathrm{pr}}\left(\Psi_{N}\left(\mu, t^{K-k+k^{\prime}}\right) ; \mu, t^{k^{\prime}}\right) \Delta t, \quad \forall \mu \in \mathcal{D}, \forall k \in \mathbb{K},
$$

respectively. The error in the output of interest is then bounded by

$$
\left|s\left(\mu, t^{k}\right)-s_{N}\left(\mu, t^{k}\right)\right| \leq \Delta^{s}\left(\mu, t^{k}\right), \quad \forall \mu \in \mathcal{D}, \forall k \in \mathbb{K}
$$

where the output bound $\Delta^{s}\left(\mu, t^{k}\right)$ is defined as

$$
\Delta^{s}\left(\mu, t^{k}\right) \equiv \Delta_{N_{\mathrm{pr}}}^{\mathrm{pr}}\left(\mu, t^{k}\right) \Delta_{N_{\mathrm{du}}}^{\mathrm{du}}\left(\mu, t^{K-k+1}\right),
$$

and $\Delta_{N_{\mathrm{pr}}}^{\mathrm{pr}}\left(\mu, t^{k}\right)$ and $\Delta_{N_{\mathrm{du}}}^{\mathrm{du}}\left(\mu, t^{k}\right)$ are defined in Propositions 4.1 and 4.3 , respectively.

Proof. To begin, we recall the definition of the dual problem for the output at time $t^{L}, L \in \mathbb{K}$, given by

$$
m\left(v, \psi_{L}\left(\mu, t^{k}\right) ; \mu\right)+\Delta t a\left(v, \psi_{L}\left(\mu, t^{k}\right) ; \mu\right)=m\left(v, \psi_{L}\left(\mu, t^{k+1}\right) ; \mu\right), \quad \forall v \in Y,(K \geq) L \geq k \geq 1
$$

with final condition $m\left(v, \psi_{L}\left(\mu, t^{L+1}\right) ; \mu\right) \equiv \ell(v), \forall v \in Y$. We now choose $v=e^{\operatorname{pr}}\left(\mu, t^{k}\right)=y\left(\mu, t^{k}\right)-y_{N}\left(\mu, t^{k}\right)$ in (63) and sum from $k=1$ to $L$, to obtain

$$
\sum_{k^{\prime}=1}^{L} m\left(e^{\mathrm{pr}}\left(\mu, t^{k^{\prime}}\right), \psi_{L}\left(\mu, t^{k^{\prime}}\right)-\psi_{L}\left(\mu, t^{k^{\prime}+1}\right) ; \mu\right)+\sum_{k^{\prime}=1}^{L} \Delta t a\left(e^{\mathrm{pr}}\left(\mu, t^{k^{\prime}}\right), \psi_{L}\left(\mu, t^{k^{\prime}}\right) ; \mu\right)=0 .
$$


This equation can be rewritten in the form

$$
\begin{aligned}
\sum_{k^{\prime}=1}^{L} m\left(e^{\mathrm{pr}}\left(\mu, t^{k^{\prime}}\right)-e^{\mathrm{pr}}\left(\mu, t^{k^{\prime}-1}\right), \psi_{L}\left(\mu, t^{k^{\prime}}\right) ; \mu\right)-m\left(e^{\mathrm{pr}}\left(\mu, t^{L}\right), \psi_{L}\left(\mu, t^{L+1}\right) ; \mu\right) & \\
& +\sum_{k^{\prime}=1}^{L} \Delta t a\left(e^{\mathrm{pr}}\left(\mu, t^{k^{\prime}}\right), \psi_{L}\left(\mu, t^{k^{\prime}}\right) ; \mu\right)=0
\end{aligned}
$$

where we used the fact that $e^{\mathrm{pr}}\left(\mu, t^{0}\right)=0$. We now note from the final condition of the dual problem that $m\left(e^{\mathrm{pr}}\left(\mu, t^{L}\right), \psi_{L}\left(\mu, t^{L+1}\right) ; \mu\right)=\ell\left(e^{\mathrm{pr}}\left(\mu, t^{L}\right)\right)$ to obtain

$$
\ell\left(e^{\mathrm{pr}}\left(\mu, t^{L}\right)\right)=\sum_{k^{\prime}=1}^{L} m\left(e^{\mathrm{pr}}\left(\mu, t^{k^{\prime}}\right)-e^{\mathrm{pr}}\left(\mu, t^{k^{\prime}-1}\right), \psi_{L}\left(\mu, t^{k^{\prime}}\right) ; \mu\right)+\sum_{k^{\prime}=1}^{L} \Delta t a\left(e^{\mathrm{pr}}\left(\mu, t^{k^{\prime}}\right), \psi_{L}\left(\mu, t^{k^{\prime}}\right) ; \mu\right) .
$$

We next choose $v=\psi_{L}\left(\mu, t^{k}\right)$ in the error equation for the primal variable, (40), and sum from $k=1$ to $L$, to find

$$
\begin{aligned}
& \sum_{k^{\prime}=1}^{L} m\left(e^{\mathrm{pr}}\left(\mu, t^{k^{\prime}}\right)-e^{\mathrm{pr}}\left(\mu, t^{k^{\prime}-1}\right), \psi_{L}\left(\mu, t^{k^{\prime}}\right) ; \mu\right)+\sum_{k^{\prime}=1}^{L} \Delta t a\left(e^{\mathrm{pr}}\left(\mu, t^{k^{\prime}}\right), \psi_{L}\left(\mu, t^{k^{\prime}}\right) ; \mu\right) \\
&=\sum_{k^{\prime}=1}^{L} R^{\mathrm{pr}}\left(\psi_{L}\left(\mu, t^{k^{\prime}}\right) ; \mu, t^{k^{\prime}}\right) \Delta t
\end{aligned}
$$

From (66) and (67) we thus obtain

$$
\begin{aligned}
\ell\left(e^{\mathrm{pr}}\left(\mu, t^{L}\right)\right) & =\sum_{k^{\prime}=1}^{L} R^{\mathrm{pr}}\left(\psi_{L}\left(\mu, t^{k^{\prime}}\right) ; \mu, t^{k^{\prime}}\right) \Delta t \\
& =\sum_{k^{\prime}=1}^{L} R^{\mathrm{pr}}\left(\Psi\left(\mu, t^{K-L+k^{\prime}}\right) ; \mu, t^{k^{\prime}}\right) \Delta t
\end{aligned}
$$

From the definition of $s\left(\mu, t^{k}\right)$ and $s_{N}\left(\mu, t^{k}\right)$, and (69) we now obtain

$$
\begin{aligned}
s\left(\mu, t^{k}\right)-s_{N}\left(\mu, t^{k}\right) & =\ell\left(e^{\mathrm{pr}}\left(\mu, t^{k}\right)\right)-\sum_{k^{\prime}=1}^{k} R^{\mathrm{pr}}\left(\Psi_{N}\left(\mu, t^{K-k+k^{\prime}}\right) ; \mu, t^{k^{\prime}}\right) \Delta t \\
& =\sum_{k^{\prime}=1}^{k} R^{\mathrm{pr}}\left(\Psi\left(\mu, t^{K-k+k^{\prime}}\right)-\Psi_{N}\left(\mu, t^{K-k+k^{\prime}}\right) ; \mu, t^{k^{\prime}}\right) \Delta t \\
& =\sum_{k^{\prime}=1}^{k} R^{\mathrm{pr}}\left(e^{\mathrm{du}}\left(\mu, t^{K-k+k^{\prime}}\right) ; \mu, t^{k^{\prime}}\right) \Delta t .
\end{aligned}
$$


Invoking (32) and the Cauchy-Schwarz inequality we arrive at

$$
\begin{aligned}
\left|s\left(\mu, t^{k}\right)-s_{N}\left(\mu, t^{k}\right)\right| & \leq \sum_{k^{\prime}=1}^{k} \varepsilon_{N_{\mathrm{pr}}}^{\mathrm{pr}}\left(\mu, t^{k^{\prime}}\right)\left\|e^{\mathrm{du}}\left(\mu, t^{K-k+k^{\prime}}\right)\right\|_{Y} \Delta t \\
& \leq\left(\sum_{k^{\prime}=1}^{k} \varepsilon_{N_{\mathrm{pr}}}^{\mathrm{pr}}\left(\mu, t^{k^{\prime}}\right)^{2} \Delta t\right)^{\frac{1}{2}}\left(\sum_{k^{\prime}=1}^{k}\left\|e^{\mathrm{du}}\left(\mu, t^{K-k+k^{\prime}}\right)\right\|_{Y}^{2} \Delta t\right)^{\frac{1}{2}} .
\end{aligned}
$$

Let us first bound the second term on the right hand side. From (7) and the fact that $\hat{\alpha}(\mu) \leq \alpha(\mu), \forall \mu \in \mathcal{D}$, we obtain

$$
\left\|e^{\mathrm{du}}\left(\mu, t^{K-k+k^{\prime}}\right)\right\|_{Y}^{2} \leq \frac{1}{\hat{\alpha}(\mu)} a\left(e^{\mathrm{du}}\left(\mu, t^{K-k+k^{\prime}}\right), e^{\mathrm{du}}\left(\mu, t^{K-k+k^{\prime}}\right) ; \mu\right), \quad \forall \mu \in \mathcal{D} .
$$

Performing the sum from $k^{\prime}=1$ to $k$ leads to

$$
\begin{aligned}
\sum_{k^{\prime}=1}^{k}\left\|e^{\mathrm{du}}\left(\mu, t^{K-k+k^{\prime}}\right)\right\|_{Y}^{2} \Delta t \leq & \frac{1}{\hat{\alpha}(\mu)} \sum_{k^{\prime}=1}^{k} a\left(e^{\mathrm{du}}\left(\mu, t^{K-k+k^{\prime}}\right), e^{\mathrm{du}}\left(\mu, t^{K-k+k^{\prime}}\right) ; \mu\right) \Delta t \\
= & \frac{1}{\hat{\alpha}(\mu)} \sum_{k^{\prime}=K-k+1}^{K} a\left(e^{\mathrm{du}}\left(\mu, t^{k^{\prime}}\right), e^{\mathrm{du}}\left(\mu, t^{k^{\prime}}\right) ; \mu\right) \Delta t \\
\leq & \frac{1}{\hat{\alpha}(\mu)}\left(\sum_{k^{\prime}=K-k+1}^{K} a\left(e^{\mathrm{du}}\left(\mu, t^{k^{\prime}}\right), e^{\mathrm{du}}\left(\mu, t^{k^{\prime}}\right) ; \mu\right) \Delta t\right. \\
& \left.+m\left(e^{\mathrm{du}}\left(\mu, t^{K-k+1}\right), e^{\mathrm{du}}\left(\mu, t^{K-k+1}\right) ; \mu\right)\right) \\
= & \frac{1}{\hat{\alpha}(\mu)}\left(\left|\left\|e^{\mathrm{du}}\left(\mu, t^{K-k+1}\right) \mid\right\|^{\mathrm{du}}\right)^{2},\right.
\end{aligned}
$$

where the second inequality follows from the coercivity of $m(\cdot, \cdot ; \mu)$ and the last equality from the definition (52) of the $\|\left.|\cdot|\right|^{\text {du }}$-norm. Finally, inserting (80) into (74) and invoking (53) and (39), we obtain

$$
\left|s\left(\mu, t^{k}\right)-s_{N}\left(\mu, t^{k}\right)\right| \leq \Delta_{N_{\mathrm{pr}}}^{\mathrm{pr}}\left(\mu, t^{k}\right) \Delta_{N_{\mathrm{du}}}^{\mathrm{du}}\left(\mu, t^{K-k+1}\right),
$$

which is the result stated in Proposition 4.4.

\subsection{Computational procedure}

We now turn to the development of offline-online computational procedures for the calculation of $\Delta_{N_{\mathrm{pr}}}^{\mathrm{pr}}\left(\mu, t^{k}\right)$, $\Delta_{N_{\mathrm{du}}}^{\mathrm{du}}\left(\mu, t^{k}\right)$, and $\Delta^{s}\left(\mu, t^{k}\right)$. The necessary computations for the offline and online stages - by construction rather similar to the elliptic case [34] - are detailed in Appendix A.2. Here, we only summarize the computational costs involved.

The computational cost in the offline stage is (to leading order) $O\left(\left(N_{\mathrm{pr}, \max }+N_{\mathrm{du}, \max }\right)\left(Q_{a}+Q_{m}\right)\right)$ solutions of the underlying "truth" finite element approximation and $O\left(\left(N_{\mathrm{pr}, \max }^{2}+N_{\mathrm{du}, \max }^{2}\right)\left(Q_{a}^{2}+Q_{a} Q_{m}+Q_{m}^{2}\right)\right) \mathcal{N}$-inner products; the storage requirement is $O\left(\left(N_{\mathrm{pr}, \max }^{2}+N_{\mathrm{du}, \max }^{2}\right)\left(Q_{a}^{2}+Q_{a} Q_{m}+Q_{m}^{2}\right)\right)$. In the online stage - given a new parameter value $\mu$ and associated reduced-basis solutions $\underline{y}_{N}\left(\mu, t^{k}\right)$ and $\underline{\Psi}_{N}\left(\mu, t^{k}\right), \forall k \in \mathbb{K}$ - the computational cost to evaluate $\Delta^{s}\left(\mu, t^{k}\right), \forall k \in \mathbb{K}$, is $O\left(K\left(N_{\mathrm{pr}}^{2}+N_{\mathrm{du}}^{2}\right)\left(Q_{a}^{2}+Q_{a} Q_{m}+Q_{m}^{2}\right)\right)$. Thus, all online calculations needed are independent of $\mathcal{N}$. 


\section{AdAptive SAMPling PROCEDURE}

Our error estimation procedures not only allow us to determine the accuracy of the output estimate but also to pursue a more rational construction of the sampling set $S_{N_{\mathrm{pr}}}^{\mathrm{pr}}$ (and $S_{N_{\mathrm{du}}}^{\mathrm{du}}$ ) and associated reduced-basis space $W_{N_{\mathrm{pr}}}^{\mathrm{pr}}$ (and $W_{N_{\mathrm{du}}}^{\mathrm{du}}$ ). The crucial point is that the error bound $\Delta_{N_{\mathrm{pr}}}^{\mathrm{pr}}\left(\mu, t^{k}\right)$ (respectively, $\left.\Delta_{N_{\mathrm{du}}}^{\mathrm{du}}\left(\mu, t^{k}\right)\right)$ is an accurate surrogate for the true error $\left\|\mid y\left(\mu, t^{k}\right)-y_{N}\left(\mu, t^{k}\right)\right\|^{\mid \operatorname{pr}}\left(\right.$ respectively, $\left.\left\|\mid \Psi\left(\mu, t^{k}\right)-\Psi_{N}\left(\mu, t^{k}\right)\right\|^{\text {du }}\right)$ that can be very efficiently calculated in the limit of many queries. We may thus perform an exhaustive search over the parameter-time space to find the best sample sets $S_{N_{\mathrm{pr}}}^{\mathrm{pr}}$ (and $S_{N_{\mathrm{du}}}^{\mathrm{du}}$ ): in essence, a snapshot procedure in which only the snapshots retained must actually be evaluated.

The sampling procedure for the primal and dual problem is very similar; we thus focus only on the primal problem. Also recall that the control input sequence $u\left(t^{k}\right)$ is assumed to be known - either a prescribed function or the impulse (see Sect. 2.2).

\subsection{Greedy algorithm}

To begin, we assume that we are given a sample set $S_{N_{\mathrm{pr}}}^{\mathrm{pr}}$ and associated reduced-basis space $W_{N_{\mathrm{pr}}}^{\mathrm{pr}}$. We then choose the next sampling point based on the following two steps: first, we search in parameter space and select the parameter value $\mu^{*}$ for which $\Delta_{N_{\mathrm{pr}}}^{\mathrm{pr}}\left(\mu, t^{K}\right)$ is maximized, ${ }^{3}$

$$
\mu^{*}=\arg \max _{\mu \in \Xi_{F}} \Delta_{N_{\mathrm{pr}}}^{\mathrm{pr}}\left(\mu, t^{K}\right)
$$

we then select the timestep $t^{k^{*}}$ for which the temporal rate of change of $\Delta_{N_{\mathrm{pr}}}^{\mathrm{pr}}\left(\mu, t^{k}\right)$ is largest,

$$
t^{k^{*}}=\arg \max _{t^{k} \in \mathbb{I}}\left(\Delta_{N_{\mathrm{pr}}}^{\mathrm{pr}}\left(\mu, t^{k}\right)-\Delta_{N_{\mathrm{pr}}}^{\mathrm{pr}}\left(\mu, t^{k-1}\right)\right)
$$

Here, $\Xi_{F} \subset(\mathcal{D})^{n_{F}}$ is a random parameter test sample of size $n_{F} ;$ since the marginal cost to evaluate $\Delta_{N_{\mathrm{pr}}}^{\mathrm{pr}}\left(\mu, t^{K}\right)$ is small, the random sample can be very large, i.e., $n_{F} \gg 1$. We then append $\tilde{\mu}^{*}=\left(\mu^{*}, t^{k^{*}}\right)$ to $S_{N_{\mathrm{pr}}}^{\mathrm{pr}}$ to form $S_{N_{\mathrm{pr}}+1}^{\mathrm{pr}}$, and hence $W_{N_{\mathrm{pr}}+1}^{\mathrm{pr}}$, and update the reduced-basis approximation and error estimation procedure accordingly. We repeat this process until the maximum error bound at the final time $t^{K}$ over $\Xi_{F}$ is less than a desired (most stringent anticipated) error tolerance $\epsilon_{\text {tol,min }}$ : this determines $N_{\text {pr,max }}$.

We note that our sample selection process is not truly optimal: given the prescribed error tolerance $\epsilon_{\text {tol,min }}$, there are undoubtedly parameter samples with fewer than $N_{\mathrm{pr} \text {, max }}$ points that suffice. Unfortunately, the latter can only be identified by prohibitively (combinatorially) expensive calculation, and thus we must resort to heuristic approaches. Our particular heuristic, described above, is of the "greedy" [9] variety: we focus on just the next sample point and just the currently largest error with no regard to more global objectives. In actual practice, as we shall see in Section 6, this carpe diem philosophy indeed leads to good samples; but we are not able to characterize the degree of sub-optimality relative to truly optimal samples.

We elaborate on three refinements. First, we invoke a normalized error bound for the sampling procedure to avoid dependence on the magnitude of the forcing term (the control input): in particular, we normalize with respect to $\left\|y_{N}\left(\mu, t^{K}\right)\right\|^{\mathrm{pr}}$, which can be calculated online in only $O\left(K N_{\mathrm{pr}}^{2}\right)$ operations. Second, we are careful to orthonormalize the basis functions $\zeta_{n}^{\mathrm{pr}}$ with respect to the $(\cdot, \cdot)_{Y}$ inner product by (say) Gram-Schmidt: this guarantees, for example, that the condition number of the reduced-basis matrix $A_{N}(\mu)$ is bounded from above by $\frac{\gamma_{0}}{\alpha_{0}}$ for all $N$. Third, as regards initialization, we simply set $\mu_{1}=\mu_{\min }$ and choose $\zeta_{1}^{\mathrm{pr}}=y\left(\mu_{1}, t^{k}\right) \neq 0$ for some small $k$, i.e., we select $\zeta_{1}^{\mathrm{pr}}=y\left(\mu_{1}, t^{1}\right)$ for $u\left(t^{1}\right) \neq 0$. This choice has a simple justification: the adaptive sampling procedure is likely to select samples corresponding to transient behaviour which, in most cases - and certainly for the impulse input - occurs during the first few timesteps (also see the numerical results in Fig. 4).

3 Note that $\Delta_{N_{\mathrm{pr}}}^{\mathrm{pr}}\left(\mu, t^{k}\right)$ is a nondecreasing sequence in $k$ and the maximum therefore always occurs at $k=K$. 


\subsection{Extensions}

The extension of the adaptive procedure to the case of multiple control inputs is straightforward. If the control inputs are given, the sampling algorithm can directly be applied; however, if the control inputs are unknown, e.g., in the optimal control context, we can simply adjust the impulse approach. We begin with an impulse in the first control input - all other control inputs are set to zero - and generate the basis using the standard algorithm. When the adaptive procedure terminates, we set the first control input to zero and the second control input to the impulse and restart the adaptive sampling - initialized to the already existing sample set $S_{N_{\mathrm{pr}}}^{\mathrm{pr}}$ and associated reduced-basis space $W_{N_{\mathrm{pr}}}^{\mathrm{pr}}$. In effect, the multiple control input scenario simply adds an "outer loop" to the standard algorithm.

We may also consider nonzero initial conditions. In the case of a parameter-independent nonzero initial condition, we simply set $\zeta_{1}^{\mathrm{pr}}=y_{0}$ and apply the standard algorithm. For (affinely) parameter-dependent initial conditions $y_{0}(\mu)$ we may write

$$
y_{0}(\mu)=\sum_{q=1}^{Q_{y}} \Theta_{y}^{q}(\mu) y_{0}^{q}, \quad \forall \mu \in \mathcal{D},
$$

where the $y_{0}^{q} \in Y, 1 \leq q \leq Q_{y}$, are given members of $Y$; only the functions $\Theta_{y}^{q}(\mu): \mathcal{D} \rightarrow \boldsymbol{R}, 1 \leq q \leq Q_{y}$ depend on $\mu$. In this case we initialize $W_{N_{\mathrm{pr}}}^{\mathrm{pr}}$ to $\operatorname{span}_{1 \leq q \leq Q_{y}}\left\{y_{0}^{q}\right\}$, and then apply the standard sampling algorithm of Section 5.1 (with initial condition $y_{0}(\mu)$ ). In both these cases we retain the condition $e^{\operatorname{pr}}\left(\mu, t^{0}\right)=0$.

Note that the case of multiple control inputs with nonzero initial conditions is a straightforward combination of the previous two cases. We first generate a reduced-basis for the nonzero initial condition (with zero control input); given this basis, we then further adapt to the control inputs using the impulse approach (for zero initial condition).

\section{Numerical Results}

We now turn to a particular numerical example related to transient heat conduction. We consider the design of a heat shield, one segment of which is shown in Figure 1. The domain $\Omega$, a typical point of which is $\left(x_{1}, x_{2}\right)$, is thus given by $\Omega \equiv\{[0,10] \times[0,4]\} \backslash\{(] 1,3[\times] 1,3[) \cup(] 4,6[\times] 1,3[) \cup(] 7,9[\times] 1,3[)\}$. The left boundary, $\partial \Omega_{\text {out }}$ $\left(x_{1}=0\right)$, is exposed to a hot temperature (here normalized to unity) for $\left.\left.t \in\right] 0, t_{f}\right]$; the right boundary as well as the top and bottom boundaries are insulated. The internal boundaries $\partial \Omega_{\text {in }}$ - corresponding to the surfaces of the three square cooling channels $] 1,3[\times] 1,3[] 4,,6[\times] 1,3[$, and $] 7,9[\times] 1,3[$ - are exposed to a (normalized) zero-temperature air flow. The (non-dimensionalized) heat transfer coefficients for the non-insulated boundaries $\partial \Omega_{\text {out }}$ and $\partial \Omega_{\text {in }}$ are given by the Biot numbers $\mathrm{Bi}_{\text {out }}$ and $\mathrm{Bi}_{\text {in }}$, respectively. Our input parameter is hence $\mu \equiv\left(\mu_{(1)}, \mu_{(2)}\right) \equiv\left(\mathrm{Bi}_{\text {out }}, \mathrm{Bi}_{\text {in }}\right) \in \mathcal{D} \equiv[0.01,0.5] \times[0.001,0.1] \subset \boldsymbol{R}^{P=2}$. Our output is the average temperature of the structure, which serves as a surrogate for the maximum possible temperature of the (to-be-protected) right boundary for $t \in[0, \infty[$.

The underlying partial differential equation is the heat (diffusion) equation. The (appropriately non-dimensionalized) governing equation for the temperature $y\left(\mu, t^{k}\right) \in Y$ is thus (3), where $Y \subset Y^{\mathrm{e}} \equiv H^{1}(\Omega)$ is a linear finite element truth approximation subspace of dimension (exploiting symmetry) $\mathcal{N}=1396$. The bilinear and linear forms are given by $m(w, v ; \mu) \equiv \int_{\Omega} w v, a(w, v ; \mu) \equiv \int_{\Omega} \nabla w \nabla v+\mu_{(1)} \int_{\partial \Omega_{\mathrm{out}}} w v+\mu_{(2)} \int_{\partial \Omega_{\mathrm{in}}} w v$, and $b(v ; \mu) \equiv \mu_{(1)} \int_{\partial \Omega_{\mathrm{out}}} v$; these forms admit obvious affine representations (9)-(11) with $Q_{m}=1, Q_{a}=3$, and $Q_{b}=1^{4}$. We also define the inner product $(w, v)_{Y} \equiv \int_{\Omega} \nabla w \nabla v+0.01 \int_{\partial \Omega_{\text {out }}} w v+0.001 \int_{\partial \Omega_{\text {in }}} w v$, corresponding to (35) for $\mu_{\text {ref }}=(0.01,0.001)$; we may hence choose $\hat{\alpha}(\mu)=1$ in $(30)$. The output can be written in the form $(4), s\left(\mu, t^{k}\right)=\ell\left(y\left(\mu, t^{k}\right)\right), \forall k \in \mathbb{K}$, where $\ell(v)=|\Omega|^{-1} \int_{\Omega} v$ is clearly a very smooth functional. We shall consider the time interval $\bar{I}=[0,20]$ and a timestep $\Delta t=0.2$; we thus have $K=100$.

\footnotetext{
${ }^{4}$ Note that the bilinear form $m$ happens to be parameter-independent in this example, and thus $e^{\mathrm{du}}\left(\mu, t^{K+1}\right)=0$ here. We thus have no (computational) need for $(\cdot, \cdot)_{X}$.
} 


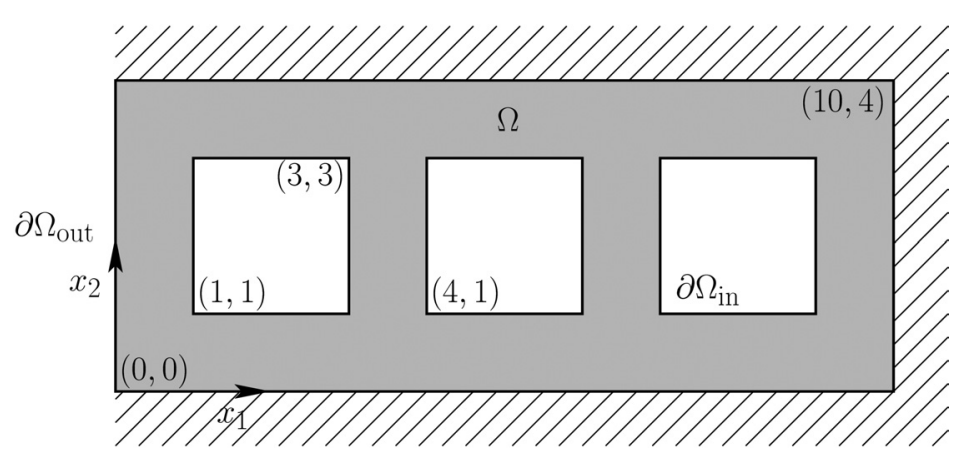

Figure 1. One segment of the heat shield.

In Figures 2 and 3 we show the temperature variation over the heat shield at different points in time and for different parameter combinations. We first note that for larger values of $\mu_{(1)}$ the temperature is, overall, much higher than for smaller values of $\mu_{(1)}$. Also, for larger values of $\mu_{(2)}$ more heat is removed through the first cooling channel; for smaller values of $\mu_{(2)}$, however, the heat penetrates deeper into the structure and the temperature tends to be higher and more uniform over the heat shield.

Before discussing the convergence properties we present numerical results for our adaptive sampling procedure. For purposes of illustration, we construct a reduced-basis space for the (one-)parameter set $\mathcal{D}_{1} \equiv[0.01] \times$ $[0.001,0.1]$, i.e., we assume $\mu_{(1)}=0.01$ is fixed. We initialize the procedure with $S_{1}^{\mathrm{pr}}=\left(\mu_{(2), \min }=0.001, t^{1}\right)$ and set the desired error tolerance (for the primal energy norm) to $\epsilon_{\text {tol,min }}=1 \mathrm{E}-3$. We plot and tabulate the resulting sample set $S_{N_{\mathrm{pr}}}^{\mathrm{pr}}$ in $\mu_{(2)}-t^{k}$ space in Figure 4 - we need $N_{\mathrm{pr}}=15$ basis functions to obtain the desired accuracy. We note that for this problem the adaptive sampling procedure selects all the samples on the $\mu_{(2)}=0.001$ axis before selecting any other samples. Also, samples taken from only near the extreme parameter values (minimum and maximum) in $\mathcal{D}_{1}$ are sufficient to guarantee the desired tolerance everywhere in $\mathcal{D}_{1}$; in general, this is not the case.

We now present convergence results for the full two-parameter numerical example. The primal and dual samples in $\tilde{\mathcal{D}}=\mathcal{D} \times \mathbb{I}$ are constructed according to the adaptive sampling procedure in Section 5; we obtain $N_{\mathrm{pr}, \max }=22$ and $N_{\mathrm{du}, \max }=21$ for $\epsilon_{\mathrm{tol}, \min }=1 \mathrm{E}-3$. (We do not consider here optimization of the primal and dual effort given desired output accuracies.) We first define the effectivity associated to the primal and dual error bounds as

$$
\eta^{\mathrm{pr}}\left(\mu, t^{k}\right) \equiv \frac{\Delta_{N_{\mathrm{pr}}}^{\mathrm{pr}}\left(\mu, t^{k}\right)}{\left\|e^{\mathrm{pr}}\left(\mu, t^{k}\right)\right\|^{\mathrm{pr}}}
$$

and

$$
\eta^{\mathrm{du}}\left(\mu, t^{k}\right) \equiv \frac{\Delta_{N_{\mathrm{du}} \mathrm{du}}\left(\mu, t^{k}\right)}{\left\|e^{\mathrm{du}}\left(\mu, t^{k}\right)\right\| \|^{\mathrm{du}}}
$$

respectively. Similarly, the effectivity for the output bound is defined as

$$
\eta^{s}\left(\mu, t^{k}\right) \equiv \frac{\Delta^{s}\left(\mu, t^{k}\right)}{\left|s\left(\mu, t^{k}\right)-s_{N}\left(\mu, t^{k}\right)\right|} .
$$

The effectivity serves as a measure of rigour and sharpness of the error bounds: we have $\eta^{\mathrm{pr}}\left(\mu, t^{k}\right) \geq 1, \forall \mu \in \mathcal{D}$, since $\Delta^{\operatorname{pr}}\left(\mu, t^{k}\right)$ is a true upper bound to the error in the $\||\cdot|||^{\mathrm{pr}}$-norm; and ideally we would like $\eta^{\mathrm{pr}}\left(\mu, t^{k}\right) \approx 1$, $\forall \mu \in \mathcal{D}$, so as to obtain a sharp bound for the error. (Similar arguments apply to the dual and to the output.)

In Table 1 we present, as a function of $N_{\mathrm{pr}}\left(=N_{\mathrm{du}}\right), \Delta_{\text {max,rel }}^{\mathrm{pr}}, \bar{\eta}^{\mathrm{pr}}, \Delta_{\text {max }, \text { rel }}^{s}$, and $\bar{\eta}^{s}: \Delta_{\text {max,rel }}^{\mathrm{pr}}$ is the maximum over $\Xi_{\text {Test }}$ of $\Delta_{N_{\mathrm{pr}}}^{\mathrm{pr}}\left(\mu, t^{K}\right) /\left\|y_{N}\left(\mu_{y}, t^{K}\right) \mid\right\|, \bar{\eta}^{\mathrm{pr}}$ is the average over $\Xi_{\text {Test }} \times \mathbb{I}$ of $\Delta_{N_{\mathrm{pr}}}^{\mathrm{pr}}\left(\mu, t^{k}\right) / \| y\left(\mu, t^{k}\right)-$ $y_{N}\left(\mu, t^{k}\right)|| \mid, \Delta_{\text {max,rel }}^{s}$ is the maximum over $\Xi_{\text {Test }}$ of $\Delta^{s}\left(\mu, t^{K}\right) /\left|s_{N}\left(\mu_{s}, t^{K}\right)\right|$, and $\bar{\eta}^{s}$ is the average over $\Xi_{\text {Test }}$ 

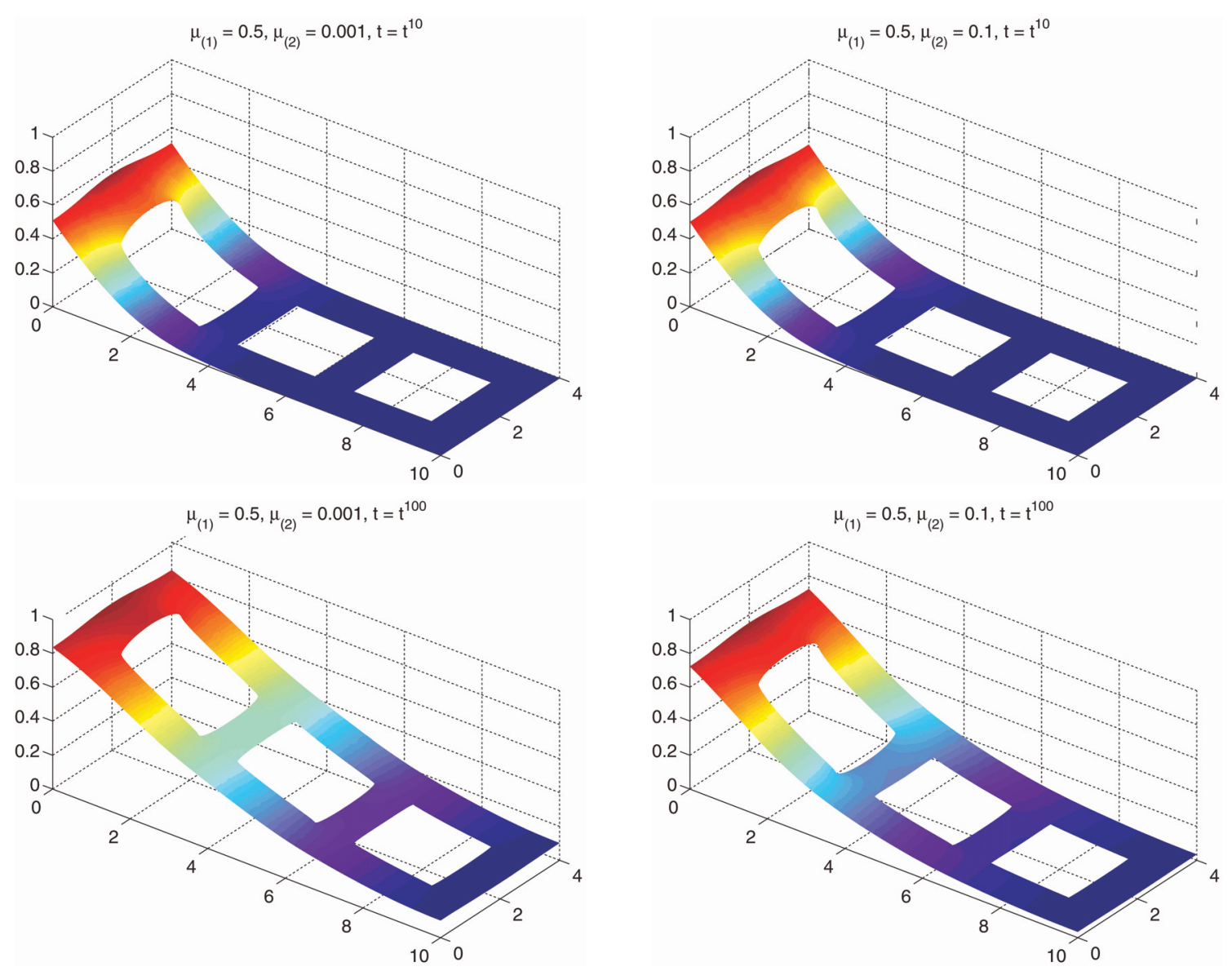

(a)

(b)

Figure 2. Temperature in the heat shield at $t=t^{10}=2$ and $t=t^{100}=20$ over the domain $\Omega$ for (a) $\mu=(0.5,0.001)$ and (b) $\mu=(0.5,0.1)$.

of $\Delta^{s}\left(\mu, t_{\eta}(\mu)\right) /\left|s\left(\mu, t_{\eta}(\mu)\right)-s_{N}\left(\mu, t_{\eta}(\mu)\right)\right|$. Here $\Xi_{\text {Test }} \in(\mathcal{D})^{400}$ is a random input sample of size $400 ; \mu_{y} \equiv$ $\arg \max _{\mu \in \Xi_{\text {Test }}}||\left|y_{N_{\max }}\left(\mu, t^{K}\right)\right|||, \mu_{s} \equiv \arg \max _{\mu \in \Xi_{\text {Test }}}\left|s_{N_{\max }}\left(\mu, t^{K}\right)\right|$ (note the output grows with time), and $t_{\eta}(\mu) \equiv \arg \max _{t^{k} \in \mathbb{I}}\left|s\left(\mu, t^{k}\right)-s_{N}\left(\mu, t^{k}\right)\right|$. We observe very rapid convergence of the reduced-basis approximation. Furthermore, as we may expect, $\Delta^{s}\left(\mu, t^{k}\right)$ converges roughly as the square of $\Delta_{N_{\mathrm{pr}}}^{\mathrm{pr}}\left(\mu, t^{k}\right)$; we see that for only $N_{\mathrm{pr}}=N_{\mathrm{du}}=8$ the error in the output is less than one percent. Also, the effectivities are very good: $O(1)$ for the primal error bound, and $O(10)$ for the output bound; note the latter are worse than the former as our bound cannot take into account any correlation between the primal and dual error. (We do not at present have good a priori upper bounds for the effectivities; see [34] for treatment of the elliptic case.)

In Table 2 we present, as a function of $N_{\mathrm{pr}}\left(=N_{\mathrm{du}}\right)$, the online computational times to calculate $s_{N}\left(\mu, t^{k}\right)$ and $\Delta^{s}\left(\mu, t^{k}\right), \forall k \in \mathbb{K}$. The values are normalized with respect to the computational time for the direct calculation of the truth approximation output $s\left(\mu, t^{k}\right)=\ell\left(u\left(\mu, t^{k}\right)\right), \forall k \in \mathbb{K}$. We note that even for the largest value of $N_{\mathrm{pr}}\left(=N_{\mathrm{du}}\right)$ the calculation of $s_{N}\left(\mu, t^{k}\right)$ and $\Delta^{s}\left(\mu, t^{k}\right)$ is approximately 100 times faster than the direct calculation of $s\left(\mu, t^{k}\right)$. (The growth with $N_{\mathrm{pr}}$ is less than expected due to memory-access issues.) We emphasize that the reduced-basis entry does not include the extensive offline computations - and is thus only meaningful in the real-time or many-query contexts. 

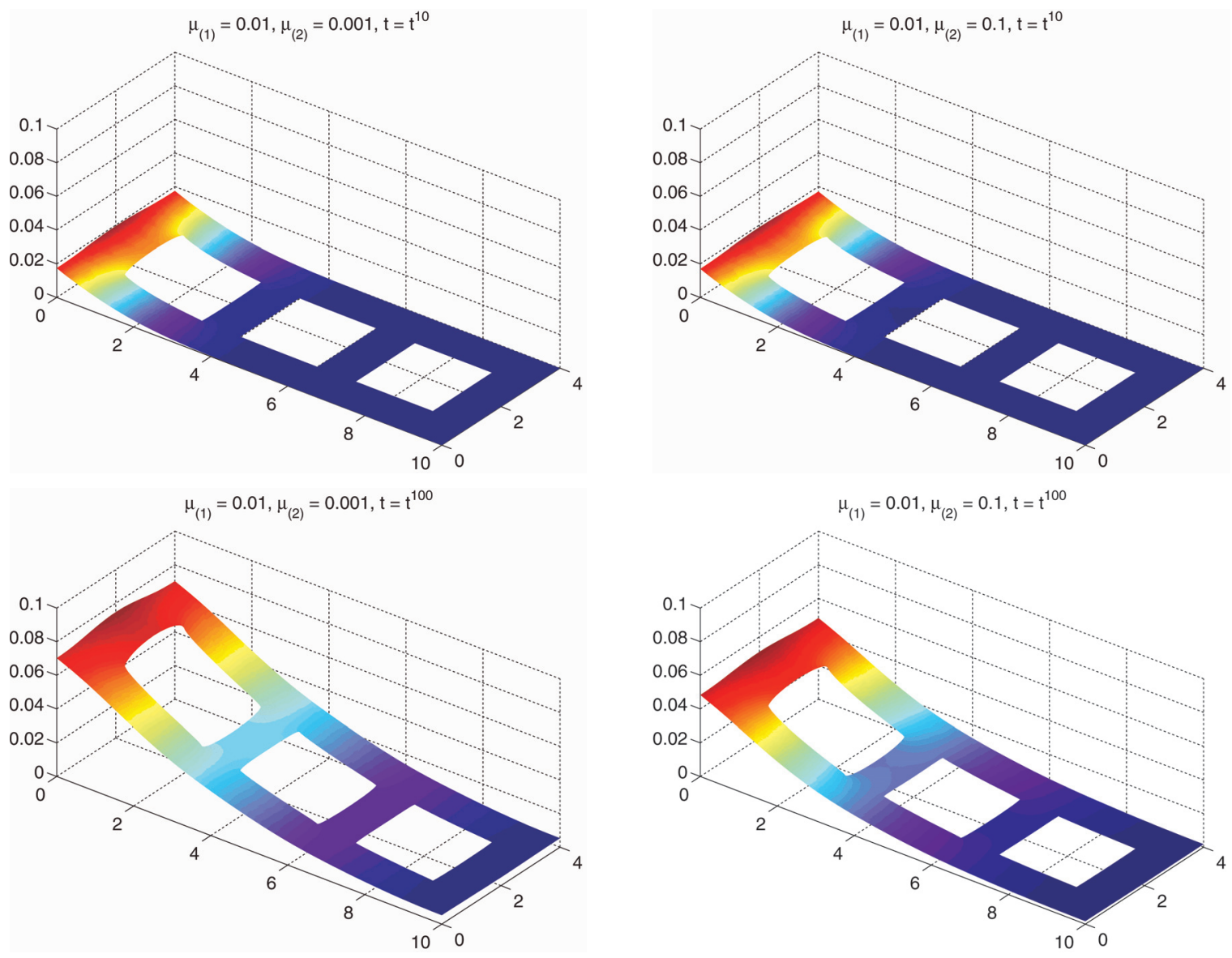

(a)

(b)

FiguRE 3. Temperature in the heat shield at $t=t^{10}=2$ and $t=t^{100}=20$ over the domain $\Omega$ for (a) $\mu=(0.01,0.001)$ and (b) $\mu=(0.01,0.1)$.

We can now define lower and upper output bounds

$$
s_{N}^{-}\left(\mu, t^{k}\right) \equiv s_{N}\left(\mu, t^{k}\right)-\Delta^{s}\left(\mu, t^{k}\right) \leq s\left(\mu, t^{k}\right) \leq s_{N}\left(\mu, t^{k}\right)+\Delta^{s}\left(\mu, t^{k}\right) \equiv s_{N}^{+}\left(\mu, t^{k}\right) .
$$

We know that $s_{N}^{+}\left(\mu, t^{k}\right)$ (respectively, $s_{N}^{-}\left(\mu, t^{k}\right)$ ) are certifiably upper (respectively, lower) bounds for the true output $s\left(\mu, t^{k}\right)$ - see Proposition 4.4; that these bounds are accurate - see Table 1; and that these bounds may be evaluated very fast online - see Table 2 . The bounds may thus serve to ensure a feasible $\operatorname{design}^{5}$, a "good" design, and a fast design process or real-time decision [29].

\footnotetext{
${ }^{5}$ For example, to honor an optimal-control constraint of the form $s\left(\mu, t^{k}\right) \leq T_{\max }$ we may conservatively impose $s_{N}^{+}\left(\mu, t^{k}\right) \leq$ $T_{\max }$.
} 


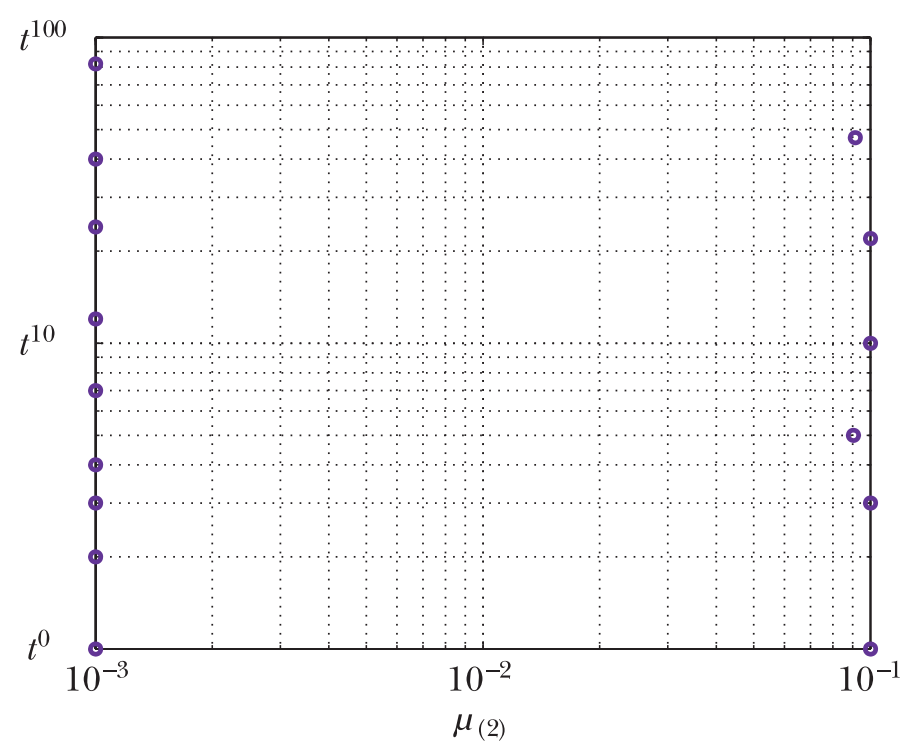

\begin{tabular}{|c||c|c|}
\hline$n$ & $\mu_{n}^{\mathrm{pr}}$ & $k_{n}^{\mathrm{pr}}$ \\
\hline 1 & 0.001 & 1 \\
2 & 0.001 & 2 \\
3 & 0.001 & 3 \\
4 & 0.001 & 4 \\
5 & 0.001 & 7 \\
6 & 0.001 & 12 \\
7 & 0.001 & 24 \\
8 & 0.001 & 40 \\
9 & 0.001 & 82 \\
10 & 0.100 & 1 \\
11 & 0.100 & 3 \\
12 & 0.100 & 10 \\
13 & 0.100 & 22 \\
14 & 0.090 & 5 \\
15 & 0.091 & 47 \\
\hline
\end{tabular}

Figure 4. Sample set $S_{N_{\mathrm{pr}}}^{\mathrm{pr}}$ for $\mathcal{D}_{1} \equiv[0.01] \times[0.001,0.1]$ and $N_{\mathrm{pr}}=15$.

TABLE 1. Convergence rate and effectivities: $N_{\mathrm{pr}}=N_{\mathrm{du}}$.

\begin{tabular}{|r||c|c|c|c|}
\hline$N_{\mathrm{pr}}$ & $\Delta_{\text {max }, \mathrm{rel}}^{\mathrm{pr}}$ & $\bar{\eta}^{\mathrm{pr}}$ & $\Delta_{\text {max }, \mathrm{rel}}^{s}$ & $\bar{\eta}^{s}$ \\
\hline 4 & $1.6 \mathrm{E}-00$ & 5.44 & $1.6 \mathrm{E}-00$ & 95.63 \\
8 & $6.3 \mathrm{E}-02$ & 1.55 & $6.7 \mathrm{E}-03$ & 30.92 \\
12 & $1.0 \mathrm{E}-02$ & 1.03 & $2.6 \mathrm{E}-04$ & 8.43 \\
16 & $3.2 \mathrm{E}-03$ & 1.02 & $1.5 \mathrm{E}-05$ & 11.45 \\
20 & $8.8 \mathrm{E}-04$ & 1.01 & $1.1 \mathrm{E}-06$ & 17.43 \\
\hline
\end{tabular}


TABLE 2. Online computational times (normalized with respect to the time to solve for $\left.s\left(\mu, t^{k}\right), \forall k \in \mathbb{K}\right)$.

\begin{tabular}{|r||c|c|c|}
\hline$N_{\mathrm{pr}}$ & $s_{N}\left(\mu, t^{k}\right), \forall k \in \mathbb{K}$ & $\Delta^{s}\left(\mu, t^{k}\right), \forall k \in \mathbb{K}$ & $s\left(\mu, t^{k}\right), \forall k \in \mathbb{K}$ \\
\hline 4 & $3.3 \mathrm{E}-03$ & $3.2 \mathrm{E}-03$ & 1 \\
8 & $4.1 \mathrm{E}-03$ & $3.3 \mathrm{E}-03$ & 1 \\
12 & $4.9 \mathrm{E}-03$ & $3.4 \mathrm{E}-03$ & 1 \\
16 & $5.6 \mathrm{E}-03$ & $3.5 \mathrm{E}-03$ & 1 \\
20 & $6.5 \mathrm{E}-03$ & $3.6 \mathrm{E}-03$ & 1 \\
\hline
\end{tabular}

\section{A. Appendix: Offline-online computational procedure}

\section{A.1. Reduced-basis approximation}

We summarize here the reduced-basis approximations and necessary quantities for the dual problem and the output estimate (for the primal problem, see Section 3.2).

For the dual problem we define $\underline{\Psi}_{N}\left(\mu, t^{k}\right)=\left[\Psi_{N 1}\left(\mu, t^{k}\right) \Psi_{N 2}\left(\mu, t^{k}\right) \ldots \Psi_{N N_{\mathrm{du}}}\left(\mu, t^{k}\right)\right]^{T}$ and obtain from (18) that

where

$$
\left(M_{N}^{\mathrm{du}}(\mu)+\Delta t A_{N}^{\mathrm{du}}(\mu)\right) \underline{\Psi}_{N}\left(\mu, t^{k}\right)=M_{N}^{\mathrm{du}}(\mu) \underline{\Psi}_{N}\left(\mu, t^{k+1}\right), \quad \forall k \in \mathbb{K},
$$

$$
M_{N}^{\mathrm{du}}(\mu)=\sum_{q=1}^{Q_{m}} \Theta_{m}^{q}(\mu) M_{N}^{\mathrm{du} q}, \quad \text { and } A_{N}^{\mathrm{du}}(\mu)=\sum_{q=1}^{Q_{a}} \Theta_{a}^{q}(\mu) A_{N}^{\mathrm{du} q},
$$

with entries

$$
\begin{aligned}
M_{N i, j}^{\mathrm{du} q}=m^{q}\left(\zeta_{i}^{\mathrm{du}}, \zeta_{j}^{\mathrm{du}}\right), 1 \leq i, j \leq N_{\mathrm{du}, \max }, 1 \leq q \leq Q_{m} ; \\
A_{N i, j}^{\mathrm{du} q}=a^{q}\left(\zeta_{i}^{\mathrm{du}}, \zeta_{j}^{\mathrm{du}}\right), \quad 1 \leq i, j \leq N_{\mathrm{du}, \max }, 1 \leq q \leq Q_{a} ; \text { and } \\
L_{N i}^{\mathrm{du}}=\ell\left(\zeta_{i}^{\mathrm{du}}\right), \quad 1 \leq i \leq N_{\mathrm{du}, \max } .
\end{aligned}
$$

Note that $\underline{\Psi}_{N}\left(\mu, t^{K+1}\right)$ is calculated from $M_{N}^{\mathrm{du}}(\mu) \underline{\Psi}_{N}\left(\mu, t^{K+1}\right)=L_{N}^{\mathrm{du}}$.

Finally, we evaluate the output estimate, $\forall k \in \mathbb{K}$, from

$$
\begin{aligned}
& s_{N}\left(\mu, t^{k}\right)=L_{N}^{\mathrm{pr} T} \underline{y}_{N}\left(\mu, t^{k}\right)+\sum_{k^{\prime}=1}^{k} \underline{\Psi}_{N}^{T}\left(\mu, t^{K-k+k^{\prime}}\right) \\
&\left\{B_{N}^{\mathrm{du}}(\mu) u\left(t^{k^{\prime}}\right)-A_{N}^{\mathrm{pr}, \mathrm{du}}(\mu) \underline{y}_{N}\left(\mu, t^{k^{\prime}}\right)-\frac{1}{\Delta t} M_{N}^{\mathrm{pr}, \mathrm{du}}(\mu)\left(\underline{y}_{N}\left(\mu, t^{k^{\prime}}\right)-\underline{y}_{N}\left(\mu, t^{k^{\prime}-1}\right)\right)\right\}
\end{aligned}
$$

where

$$
M_{N}^{\mathrm{pr}, \mathrm{du}}(\mu)=\sum_{q=1}^{Q_{m}} \Theta_{m}^{q}(\mu) M_{N}^{\mathrm{pr}, \mathrm{du} q}, \quad A_{N}^{\mathrm{pr}, \mathrm{du}}(\mu)=\sum_{q=1}^{Q_{a}} \Theta_{a}^{q}(\mu) A_{N}^{\mathrm{pr}, \mathrm{du} q}, \quad \text { and } B_{N}^{\mathrm{du}}(\mu)=\sum_{q=1}^{Q_{b}} \Theta_{b}^{q}(\mu) B_{N}^{\mathrm{du} q},
$$

with entries

$$
\begin{array}{rlrl}
M_{N i, j}^{\mathrm{pr} q}=m^{q}\left(\zeta_{i}^{\mathrm{du}}, \zeta_{j}^{\mathrm{pr}}\right), & 1 \leq i \leq N_{\mathrm{du}, \max }, 1 \leq j \leq N_{\mathrm{pr}, \max }, 1 \leq q \leq Q_{m} ; \\
A_{N i, j}^{\mathrm{pr}, \mathrm{du} q}=a^{q}\left(\zeta_{i}^{\mathrm{du}}, \zeta_{j}^{\mathrm{pr}}\right), & 1 \leq i \leq N_{\mathrm{du}, \max }, 1 \leq j \leq N_{\mathrm{pr}, \max }, 1 \leq q \leq Q_{a} ; \\
B_{N i}^{\mathrm{du} q}=b^{q}\left(\zeta_{i}^{\mathrm{du}}\right), & & 1 \leq i \leq N_{\mathrm{du}, \max }, 1 \leq q \leq Q_{b} ; \text { and } \\
L_{N i}^{\mathrm{pr}}=\ell\left(\zeta_{i}^{\mathrm{pr}}\right), & & 1 \leq i \leq N_{\mathrm{pr}, \max } .
\end{array}
$$


The offline-online procedure is described in Section 3.2.

\section{A.2. A Posteriori error estimation}

In this section we discuss the calculation of the primal and dual error bound. For the primal error bound, we first note from standard duality arguments that

$$
\begin{aligned}
\varepsilon_{N_{\mathrm{pr}}}^{\mathrm{pr}}\left(\mu, t^{k}\right) & \equiv \sup _{v \in Y} \frac{R^{\mathrm{pr}}\left(v ; \mu, t^{k}\right)}{\|v\|_{Y}} \\
& =\left\|\hat{e}^{\mathrm{pr}}\left(\mu, t^{k}\right)\right\|_{Y},
\end{aligned}
$$

where $\hat{e}^{\mathrm{pr}}\left(\mu, t^{k}\right) \in Y$ is given by

$$
\left(\hat{e}^{\mathrm{pr}}\left(\mu, t^{k}\right), v\right)_{Y}=R^{\mathrm{pr}}\left(v ; \mu, t^{k}\right), \quad \forall v \in Y
$$

(97) is effectively a Poisson problem for each $t^{k} \in \mathbb{I}$.

From (21) and the affine assumptions (9)-(11) it thus follows that $\hat{e}^{\mathrm{pr}}\left(\mu, t^{k}\right)$ satisfies

$$
\begin{aligned}
\left(\hat{e}^{\mathrm{pr}}\left(\mu, t^{k}\right), v\right)_{Y}=\sum_{q=1}^{Q_{b}} \Theta_{b}^{q}(\mu) b^{q}(v) u\left(t^{k}\right) & -\sum_{n=1}^{N_{\mathrm{pr}}}\left\{\sum_{q=1}^{Q_{a}} \Theta_{a}^{q}(\mu) y_{N n}\left(\mu, t^{k}\right) a^{q}\left(\zeta_{n}^{\mathrm{pr}}, v\right)\right. \\
& \left.+\sum_{q=1}^{Q_{m}} \frac{1}{\Delta t} \Theta_{m}^{q}(\mu)\left(y_{N n}\left(\mu, t^{k}\right)-y_{N n}\left(\mu, t^{k-1}\right)\right) m^{q}\left(\zeta_{n}^{\mathrm{pr}}, v\right)\right\}, \forall v \in Y
\end{aligned}
$$

It is clear from linear superposition that we can express $\hat{e}\left(\mu, t^{k}\right)$ as

$$
\begin{aligned}
& \hat{e}^{\mathrm{pr}}(\mu)=\sum_{q=1}^{Q_{b}} \Theta_{b}^{q}(\mu) u\left(t^{k}\right) \mathcal{B}_{q}^{\mathrm{pr}}-\sum_{n=1}^{N_{\mathrm{pr}}}\left\{\sum_{q=1}^{Q_{a}} \Theta_{a}^{q}(\mu) y_{N n}\left(\mu, t^{k}\right) \mathcal{A}_{q, n}^{\mathrm{pr}}\right. \\
& \left.+\sum_{q=1}^{Q_{m}} \frac{1}{\Delta t} \Theta_{m}^{q}(\mu)\left(y_{N n}\left(\mu, t^{k}\right)-y_{N n}\left(\mu, t^{k-1}\right)\right) \mathcal{M}_{q, n}^{\mathrm{pr}}\right\},
\end{aligned}
$$

where we calculate

$$
\begin{aligned}
& \mathcal{B}_{q}^{\mathrm{pr}} \in Y \text { from }\left(\mathcal{B}_{q}^{\mathrm{pr}}, v\right)_{Y}=b^{q}(v), \quad \forall v \in Y \text { for } 1 \leq q \leq Q_{b}, \\
& \mathcal{A}_{q, n}^{\mathrm{pr}} \in Y \text { from }\left(\mathcal{A}_{q, n}^{\mathrm{pr}}, v\right)_{Y}=a^{q}\left(\zeta_{n}^{\mathrm{pr}}, v\right), \quad \forall v \in Y \text { for } 1 \leq n \leq N_{\mathrm{pr}, \max }, 1 \leq q \leq Q_{a}, \\
& \mathcal{M}_{q, n}^{\mathrm{pr}} \in Y \text { from }\left(\mathcal{M}_{q, n}^{\mathrm{pr}}, v\right)_{Y}=m^{q}\left(\zeta_{n}^{\mathrm{pr}}, v\right), \forall v \in Y \text { for } 1 \leq n \leq N_{\mathrm{pr}, \max }, 1 \leq q \leq Q_{m} \text {; }
\end{aligned}
$$

note $\mathcal{B}, \mathcal{A}$, and $\mathcal{M}$ are parameter independent. 
From (96) and (99) it follows that

$$
\begin{aligned}
\varepsilon_{N_{\mathrm{pr}}}^{\mathrm{pr}}\left(\mu, t^{k}\right)^{2}= & \sum_{q, q^{\prime}=1}^{Q_{b}} \Theta_{b}^{q}(\mu) \Theta_{b}^{q^{\prime}}(\mu) u\left(t^{k}\right) u\left(t^{k}\right) \Lambda_{q q^{\prime}}^{\mathrm{pr}, b b} \\
& +\sum_{q=1}^{Q_{b}} \sum_{n=1}^{N_{\mathrm{pr}}} \Theta_{b}^{q}(\mu) u\left(t^{k}\right)\left(\sum_{q^{\prime}=1}^{Q_{a}} \Theta_{a}^{q^{\prime}}(\mu) y_{N n}\left(\mu, t^{k}\right) \Lambda_{q q^{\prime} n}^{\mathrm{pr}, a b}\right. \\
& \left.+\sum_{q^{\prime}=1}^{Q_{m}} \Theta_{m}^{q^{\prime}}(\mu)\left(y_{N n}\left(\mu, t^{k}\right)-y_{N n}\left(\mu, t^{k-1}\right)\right) \Lambda_{q q^{\prime} n}^{\mathrm{pr}, m b}\right) \\
& +\sum_{n, n^{\prime}=1}^{N_{\mathrm{pr}}}\left\{\sum_{q, q^{\prime}=1}^{Q_{a}} \Theta_{a}^{q}(\mu) \Theta_{a}^{q^{\prime}}(\mu) y_{N n}\left(\mu, t^{k}\right) y_{N n^{\prime}}\left(\mu, t^{k}\right) \Lambda_{q n q^{\prime} n^{\prime}}^{\mathrm{pr}, a a}\right. \\
& +\sum_{q, q^{\prime}=1}^{Q_{m}} \Theta_{m}^{q}(\mu) \Theta_{m}^{q^{\prime}}(\mu)\left(y_{N n}\left(\mu, t^{k}\right)-y_{N n}\left(\mu, t^{k-1}\right)\right)\left(y_{N n^{\prime}}\left(\mu, t^{k}\right)-y_{N n^{\prime}}\left(\mu, t^{k-1}\right)\right) \Lambda_{q n q^{\prime} n^{\prime}}^{\mathrm{pr}, m m} \\
& \left.+\sum_{q=1}^{Q_{a}} \sum_{q^{\prime}=1}^{Q_{m}} \Theta_{a}^{q}(\mu) \Theta_{m}^{q^{\prime}}(\mu) y_{N n}\left(\mu, t^{k}\right)\left(y_{N n^{\prime}}\left(\mu, t^{k}\right)-y_{N n^{\prime}}\left(\mu, t^{k-1}\right)\right) \Lambda_{q n q^{\prime} n^{\prime}}^{\mathrm{pr}, a m}\right\}
\end{aligned}
$$

where the parameter-independent quantities $\Lambda^{\mathrm{pr}}$ are defined as

$$
\begin{aligned}
& \Lambda_{q q^{\prime}}^{\mathrm{pr}, b b}=\left(\mathcal{B}_{q}^{\mathrm{pr}}, \mathcal{B}_{q^{\prime}}^{\mathrm{pr}}\right)_{Y}, \quad 1 \leq q, q^{\prime} \leq Q_{b} ; \\
& \Lambda_{q q^{\prime} n}^{\mathrm{pr}, a b}=-2\left(\mathcal{B}_{q}^{\mathrm{pr}}, \mathcal{A}_{q^{\prime}, n}^{\mathrm{pr}}\right)_{Y}, \quad 1 \leq q \leq Q_{b}, 1 \leq q^{\prime} \leq Q_{a}, 1 \leq n \leq N_{\mathrm{pr}, \max } ; \\
& \Lambda_{q q^{\prime} n}^{\mathrm{pr}, m b}=-\frac{2}{\Delta t}\left(\mathcal{B}_{q}^{\mathrm{pr}}, \mathcal{M}_{q^{\prime}, n}^{\mathrm{pr}}\right)_{Y}, \quad 1 \leq q \leq Q_{b}, 1 \leq q^{\prime} \leq Q_{m}, 1 \leq n \leq N_{\mathrm{pr}, \max } ; \\
& \Lambda_{q n q^{\prime} n^{\prime}}^{\mathrm{pr}, a a}=\left(\mathcal{A}_{q, n}^{\mathrm{pr}}, \mathcal{A}_{q^{\prime}, n^{\prime}}^{\mathrm{pr}}\right)_{Y}, \quad 1 \leq q, q^{\prime} \leq Q_{a}, 1 \leq n, n^{\prime} \leq N_{\mathrm{pr}, \max } \\
& \Lambda_{q n q^{\prime} n^{\prime}}^{\mathrm{pr}, a m}=\frac{2}{\Delta t}\left(\mathcal{A}_{q, n}^{\mathrm{pr}}, \mathcal{M}_{q^{\prime}, n^{\prime}}^{\mathrm{pr}}\right)_{Y}, \quad 1 \leq q \leq Q_{a}, 1 \leq q^{\prime} \leq Q_{m}, 1 \leq n, n^{\prime} \leq N_{\mathrm{pr}, \max } ; \\
& \Lambda_{q n q^{\prime} n^{\prime}}^{\mathrm{pr}, m m}=\frac{1}{\Delta t^{2}}\left(\mathcal{M}_{q, n}^{\mathrm{pr}}, \mathcal{M}_{q^{\prime}, n^{\prime}}^{\mathrm{pr}}\right)_{Y}, 1 \leq q, q^{\prime} \leq Q_{m}, 1 \leq n, n^{\prime} \leq N_{\mathrm{pr}, \max } .
\end{aligned}
$$

The computational procedure for the dual error bound follows arguments similar to the primal error bound presented in (95)-(99). Thus, we first solve for

$$
\begin{gathered}
\mathcal{A}_{q, n}^{\mathrm{du}} \in Y \text { from }\left(\mathcal{A}_{q, n}^{\mathrm{du}}, v\right)_{Y}=a^{q}\left(\zeta_{n}^{\mathrm{du}}, v\right), \quad \forall v \in Y \text { for } 1 \leq n \leq N_{\mathrm{du}, \mathrm{max}}, 1 \leq q \leq Q_{a}, \\
\mathcal{M}_{q, n}^{\mathrm{du}} \in Y \text { from }\left(\mathcal{M}_{q, n}^{\mathrm{du}}, v\right)_{Y}=m^{q}\left(\zeta_{n}^{\mathrm{du}}, v\right), \forall v \in Y \text { for } 1 \leq n \leq N_{\mathrm{du}, \max }, 1 \leq q \leq Q_{m}
\end{gathered}
$$

and then evaluate the dual norm from

$$
\begin{aligned}
\varepsilon_{N_{\mathrm{du}}}^{\mathrm{du}}\left(\mu, t^{k}\right)^{2}= & \sum_{n, n^{\prime}=1}^{N_{\mathrm{du}}}\left\{\sum_{q, q^{\prime}=1}^{Q_{a}} \Theta_{a}^{q}(\mu) \Theta_{a}^{q^{\prime}}(\mu) \Psi_{N n}\left(\mu, t^{k}\right) \Psi_{N n^{\prime}}\left(\mu, t^{k}\right) \Lambda_{q n q^{\prime} n^{\prime}}^{\mathrm{du}, a a}\right. \\
& +\sum_{q, q^{\prime}=1}^{Q_{m}} \Theta_{m}^{q}(\mu) \Theta_{m}^{q^{\prime}}(\mu)\left(\Psi_{N n}\left(\mu, t^{k}\right)-\Psi_{N n}\left(\mu, t^{k+1}\right)\right)\left(\Psi_{N n^{\prime}}\left(\mu, t^{k}\right)-\Psi_{N n^{\prime}}\left(\mu, t^{k+1}\right)\right) \Lambda_{q n q^{\prime} n^{\prime}}^{\mathrm{du}, m m} \\
& \left.+\sum_{q=1}^{Q_{a}} \sum_{q^{\prime}=1}^{Q_{m}} \Theta_{a}^{q}(\mu) \Theta_{m}^{q^{\prime}}(\mu) \Psi_{N n}\left(\mu, t^{k}\right)\left(\Psi_{N n^{\prime}}\left(\mu, t^{k}\right)-\Psi_{N n^{\prime}}\left(\mu, t^{k+1}\right)\right) \Lambda_{q n q^{\prime} n^{\prime}}^{\mathrm{du}, a m}\right\}
\end{aligned}
$$


where the parameter-independent quantities $\Lambda^{\text {du }}$ are defined as

$$
\begin{aligned}
\Lambda_{q n q^{\prime} n^{\prime}}^{\mathrm{du}, a a} & =\left(\mathcal{A}_{q, n}^{\mathrm{du}}, \mathcal{A}_{q^{\prime}, n^{\prime}}^{\mathrm{du}}\right)_{Y}, \quad 1 \leq q, q^{\prime} \leq Q_{a}, 1 \leq n, n^{\prime} \leq N_{\mathrm{du}, \max } \\
\Lambda_{q n q^{\prime} n^{\prime}}^{\mathrm{du}, a m} & =\frac{2}{\Delta t}\left(\mathcal{A}_{q, n}^{\mathrm{du}}, \mathcal{M}_{q^{\prime}, n^{\prime}}^{\mathrm{du}}\right)_{Y}, \quad 1 \leq q \leq Q_{a}, 1 \leq q^{\prime} \leq Q_{m}, 1 \leq n, n^{\prime} \leq N_{\mathrm{du}, \max } \\
\Lambda_{q n q^{\prime} n^{\prime}}^{\mathrm{du}, m m} & =\frac{1}{\Delta t^{2}}\left(\mathcal{M}_{q, n}^{\mathrm{du}}, \mathcal{M}_{q^{\prime}, n^{\prime}}^{\mathrm{du}}\right)_{Y}, 1 \leq q, q^{\prime} \leq Q_{m}, 1 \leq n, n^{\prime} \leq N_{\mathrm{du}, \max } .
\end{aligned}
$$

Finally, for the contribution due to the error of the dual problem at the final time we first solve for

$$
\begin{aligned}
\mathcal{L}^{\Psi_{f}} & \in Y \text { from }\left(\mathcal{L}^{\Psi_{f}}, v\right)_{X}=\ell(v), \quad \forall v \in Y, \\
\mathcal{M}_{q, n}^{\Psi_{f}} & \in Y \text { from }\left(\mathcal{M}_{q, n}^{\Psi_{f}}, v\right)_{X}=m^{q}\left(\zeta_{n}^{\mathrm{du}}, v\right), \forall v \in Y \text { for } 1 \leq n \leq N_{\mathrm{du}, \max }, 1 \leq q \leq Q_{m} ;
\end{aligned}
$$

we then evaluate the dual norm from

$$
\varepsilon_{N_{\mathrm{du}}}^{\Psi_{f}}(\mu)^{2}=\Lambda^{\Psi_{f}, \ell \ell}+\sum_{n=1}^{N_{\mathrm{du}}} \sum_{q=1}^{Q_{m}} \Theta_{m}^{q}(\mu) \Psi_{N n}\left(\mu, t^{K+1}\right)\left\{\Lambda_{q n}^{\Psi_{f}, \ell m}+\sum_{n^{\prime}=1}^{N_{\mathrm{du}}} \sum_{q^{\prime}=1}^{Q_{m}} \Theta_{m}^{q^{\prime}}(\mu) \Psi_{N n^{\prime}}\left(\mu, t^{K+1}\right) \Lambda_{q n q^{\prime} n^{\prime}}^{\Psi_{f}, m m}\right\}
$$

where the parameter-independent quantities $\Lambda^{\Psi_{f}}$ are defined as

$$
\begin{aligned}
\Lambda_{f f}^{\Psi_{f}, \ell \ell} & =\left(\mathcal{L}^{\Psi_{f}}, \mathcal{L}^{\Psi}\right)_{X} \\
\Lambda_{q n}^{\Psi_{f}, \ell m} & =-2\left(\mathcal{M}_{q, n}^{\Psi_{f}}, \mathcal{L}^{\Psi_{f}}\right)_{X}, 1 \leq q \leq Q_{m}, 1 \leq n \leq N_{\mathrm{du}, \max } \\
\Lambda_{q n q^{\prime} n^{\prime}}^{\Psi_{f}, m m} & =\left(\mathcal{M}_{q, n}^{\Psi_{f}}, \mathcal{M}_{q^{\prime}, n^{\prime}}^{\Psi_{f}}\right)_{X}, \quad 1 \leq q, q^{\prime} \leq Q_{m}, 1 \leq n, n^{\prime} \leq N_{\mathrm{du}, \max } .
\end{aligned}
$$

The offline-online decomposition is now clear. In the offline stage we first compute the quantities $\mathcal{B}^{\mathrm{pr}}, \mathcal{L}^{\Psi_{f}}$, $\mathcal{A}^{\mathrm{pr}, \mathrm{du}}$, and $\mathcal{M}^{\mathrm{pr}, \mathrm{du}, \Psi_{f}}$ from (100), (103), and (106) and then evaluate the $\Lambda^{\mathrm{pr}, \mathrm{du}, \Psi_{f}}$ from (102), (105), and (108); this requires (to leading order) $O\left(\left(N_{\mathrm{pr}, \max }+N_{\mathrm{du}, \max }\right)\left(Q_{a}+Q_{m}\right)\right)$ expensive "truth" finite element solutions, and $O\left(\left(N_{\mathrm{pr}, \max }^{2}+N_{\mathrm{du}, \max }^{2}\right)\left(Q_{a}^{2}+Q_{a} Q_{m}+Q_{m}^{2}\right)\right) \mathcal{N}$-inner products. In the online stage, given a new parameter value $\mu$ and associated reduced-basis solutions $\underline{y}_{N}\left(\mu, t^{k}\right)$ and $\underline{\Psi}_{N}\left(\mu, t^{k}\right)$, we perform the sums (101), (104), and (107) and evaluate the error bound from

$$
\Delta^{s}\left(\mu, t^{k}\right)=\left(\frac{\Delta t}{\hat{\alpha}(\mu)} \sum_{k^{\prime}=1}^{k} \varepsilon_{N_{\mathrm{pr}}}^{\mathrm{pr}}\left(\mu, t^{k^{\prime}}\right)^{2}\right)^{\frac{1}{2}}\left(\frac{\Delta t}{\hat{\alpha}(\mu)} \sum_{k^{\prime}=K-k+1}^{K} \varepsilon_{N_{\mathrm{du}}}^{\mathrm{du}}\left(\mu, t^{k^{\prime}}\right)^{2}+\frac{\varepsilon_{N_{\mathrm{du}}}^{\Psi_{f}}(\mu)^{2}}{\hat{\sigma}(\mu)}\right)^{\frac{1}{2}}, \quad \forall k \in \mathbb{K} ;
$$

it directly follows that the online operation count for $\Delta^{s}\left(\mu, t^{k}\right), \forall k \in \mathbb{K}$, is $O\left(K\left(N_{\mathrm{pr}}^{2}+N_{\mathrm{du}}^{2}\right)\left(Q_{a}^{2}+Q_{a} Q_{m}+Q_{m}^{2}\right)\right)$. Thus, all requisite online calculations are independent of the dimension of the underlying "truth" finite element space, $\mathcal{N}$.

Acknowledgements. We would like to thank Professor Yvon Maday of University Paris VI for his many invaluable contributions to our work. We would also like to thank Dr Christophe Prud'homme of EPFL and Dr Simone Deparis of MIT for helpful discussions. This work was supported by DARPA and AFOSR under Grant F49620-03-1-0356, DARPA/GEAE and AFOSR under Grant F49620-03-1-0439, and the Singapore-MIT Alliance.

\section{REFERENCES}

[1] B.O. Almroth, P. Stern and F.A. Brogan, Automatic choice of global shape functions in structural analysis. AIAA J. 16 (1978) $525-528$.

[2] J.A. Atwell and B.B. King, Proper orthogonal decomposition for reduced basis feedback controllers for parabolic equations. Math. Comput. Mod. 33 (2001) 1-19. 
[3] E. Balmes, Parametric families of reduced finite element models: Theory and applications. Mech. Syst. Signal Process. 10 (1996) 381-394.

[4] E. Balsa-Canto, A.A. Alonso and J.R. Banga, Reduced-order models for nonlinear distributed process systems and their application in dynamic optimization. Indust. Engineering Chemistry Res. 43 (2004) 3353-3363.

[5] H.T. Banks and K. Kunisch, Estimation Techniques for Distributed Parameter Systems. Systems \& Control: Foundations \& Applications. Birkhäuser (1989).

[6] M. Barrault, N.C. Nguyen, Y. Maday and A.T. Patera, An "empirical interpolation" method: Application to efficient reducedbasis discretization of partial differential equations. C. R. Acad. Sci. Paris, Sér. I. 339 (2004) 667-672.

[7] A. Barrett and G. Reddien, On the reduced basis method. Z. Angew. Math. Mech. 75 (1995) 543-549.

[8] R. Becker and R. Rannacher, Weighted a posteriori error control in finite element methods. In ENUMATH 95 Proc. World Sci. Publ., Singapore (1997).

[9] D. Bertsimas and J.N. Tsitsiklis, Introduction to Linear Optimization. Athena Scientific (1997).

[10] E.A. Christensen, M. Brøns and J.N. Sørensen, Evaluation of proper orthogonal decomposition-based decomposition techniques applied to parameter-dependent nonturbulent flows. SIAM J. Sci. Comput. 21 (2000) 1419-1434.

[11] W. Desch, F. Kappel and K. Kunisch, Eds., Control and Estimation of Distributed Parameter Systems, volume 126 of International Series of Numerical Mathematics. Birkhäuser (1998).

[12] N.H. El-Farra and P.D. Christofides, Coordinating feedback and switching for control of spatially distributed processes. Comput. Chemical Engineering 28 (2004) 111-128.

[13] J.P. Fink and W.C. Rheinboldt, On the error behavior of the reduced basis technique for nonlinear finite element approximations. Z. Angew. Math. Mech. 63 (1983) 21-28.

[14] M. Grepl, Reduced-Basis Approximations for Time-Dependent Partial Differential Equations: Application to Optimal Control. Ph.D. Thesis, Massachusetts Institute of Technology (2005) (in progress).

[15] K.-H. Hoffmann, G. Leugering and F. Tröltzsch, Eds., Optimal Control of Partial Differential Equations, volume 133 of International Series of Numerical Mathematics. Birkhäuser (1998).

[16] K. Ito and S.S. Ravindran, A reduced basis method for control problems governed by PDEs, in Control and Estimation of Distributed Parameter Systems, W. Desch, F. Kappel, and K. Kunisch Eds., Birkhäuser (1998) 153-168.

[17] K. Ito and S.S. Ravindran, A reduced-order method for simulation and control of fluid flows. J. Comput. Phys. 143 (1998) $403-425$.

[18] K. Ito and S.S. Ravindran, Reduced basis method for optimal control of unsteady viscous flows. Int. J. Comput. Fluid Dyn. 15 (2001) 97-113.

[19] S. Lall, J.E. Marsden and S. Glavaski, A subspace approach to balanced truncation for model reduction of nonlinear control systems. Int. J. Robust Nonlinear Control 12 (2002) 519-535.

[20] M. Lin Lee, Estimation of the error in the reduced basis method solution of differential algebraic equation systems. SIAM J. Numer. Anal. 28 (1991) 512-528.

[21] J.L. Lions, Optimal Control of Systems Governed by Partial Differential Equations. Springer (1971).

[22] L. Machiels, Y. Maday, I.B. Oliveira, A.T. Patera and D.V. Rovas, Output bounds for reduced-basis approximations of symmetric positive definite eigenvalue problems. C. R. Acad. Sci. Paris, Sér. I 331 (2000) $153-158$.

[23] Y. Maday, A.T. Patera and D.V. Rovas, A blackbox reduced-basis output bound method for noncoercive linear problems, in Nonlinear Partial Differential Equations and Their Applications, Collège de France Seminar Volume XIV, D. Cioranescu and J.-L. Lions Eds., Elsevier Science B.V. (2002) 533-569.

[24] M. Mattingly, E.A. Bailey, A.W. Dutton, R.B. Roemer and S. Devasia, Reduced-order modeling for hyperthermia: An extended balanced-realization-based approach. IEEE Transactions on Biomedical Engineering 45 (1998) 1154-1162.

[25] M. Mattingly, R.B. Roemer and S. Devasia, Exact temperature tracking for hyperthermia: A model-based approach. IEEE Trans. Control Systems Technology 8 (2000) 979-992.

[26] B.C. Moore, Principal component analysis in linear systems: controllability, observability, and model reduction. IEEE Trans. Automat. Control 26 (1981) 17-32.

[27] D.A. Nagy, Modal representation of geometrically nonlinear behaviour by the finite element method. Comput. Structures 10 (1979) 683-688.

[28] A.K. Noor and J.M. Peters, Reduced basis technique for nonlinear analysis of structures. AIAA J. 18 (1980) $455-462$.

[29] I.B. Oliveira and A.T. Patera, Reduced-basis techniques for rapid reliable optimization of systems described by affinely parametrized coercive elliptic partial differential equations. Optim. Engineering (2005) (submitted).

[30] H.M. Park, T.Y. Yoon and O.Y. Kim, Optimal control of rapid thermal processing systems by empirical reduction of modes. Ind. Eng. Chem. Res. 38 (1999) 3964-3975.

[31] J.S. Peterson, The reduced basis method for incompressible viscous flow calculations. SIAM J. Sci. Stat. Comput. 10 (1989) $777-786$.

[32] T.A. Porsching, Estimation of the error in the reduced basis method solution of nonlinear equations. Math. Comp. 45 (1985) 487-496.

[33] T.A. Porsching and M. Lin Lee, The reduced basis method for initial value problems. SIAM J. Numer. Anal. 24 (1987) 1277-1287. 
[34] C. Prud'homme, D. Rovas, K. Veroy, Y. Maday, A.T. Patera and G. Turinici, Reliable real-time solution of parametrized partial differential equations: Reduced-basis output bound methods. J. Fluids Engineering 124 (2002) $70-80$.

[35] A. Quarteroni and A. Valli, Numerical Approximation of Partial Differential Equations. Springer, 2nd edition (1997).

[36] S.S. Ravindaran, A reduced-order approach for optimal control of fluids using proper orthogonal decomposition. Int. J. Numer. Meth. Fluids 34 (2000) 425-448.

[37] W.C. Rheinboldt, On the theory and error estimation of the reduced basis method for multi-parameter problems. Nonlinear Anal. 21 (1993) 849-858.

[38] D.V. Rovas, L. Machiels and Y. Maday, Reduced-basis output bound methods for parabolic problems. IMA J. Appl. Math. (2005) (submitted).

[39] D.V. Rovas, Reduced-Basis Output Bound Methods for Parametrized Partial Differential Equations. Ph.D. Thesis, Massachusetts Institute of Technology, Cambridge, MA (2002).

[40] L. Sirovich and M. Kirby, Low-dimensional procedure for the characterization of human faces. J. Opt. Soc. Amer. A 4 (1987) $519-524$.

[41] K. Veroy and A.T. Patera, Certified real-time solution of the parametrized steady incompressible navier-stokes equations; Rigorous reduced-basis a posteriori error bounds. Internat. J. Numer. Methods Fluids (2005) (to appear).

[42] K. Veroy, C. Prud'homme and A.T. Patera, Reduced-basis approximation of the viscous Burgers equation: Rigorous a posteriori error bounds. C. R. Acad. Sci. Paris, Sér. I 337 (2003) 619-624.

[43] K. Veroy, C. Prud'homme, D.V. Rovas and A.T. Patera, A posteriori error bounds for reduced-basis approximation of parametrized noncoercive and nonlinear elliptic partial differential equations (AIAA Paper 2003-3847), in Proceedings of the 16th AIAA Computational Fluid Dynamics Conference (June 2003).

[44] K. Veroy, D. Rovas and A.T. Patera, A Posteriori error estimation for reduced-basis approximation of parametrized elliptic coercive partial differential equations: "Convex inverse" bound conditioners. ESAIM: COCV 8 (2002) 1007-1028. Special Volume: A tribute to J.-L. Lions.

[45] K. Willcox and J. Peraire, Balanced model reduction via the proper orthogonal decomposition, in 15th AIAA Computational Fluid Dynamics Conference, AIAA (June 2001).

To access this journal online:

www.edpsciences.org 